\title{
Ground-based acoustic parametric generator impact on the atmosphere and ionosphere in an active experiment
}

\author{
Yuriy G. Rapoport ${ }^{1,2}$, Oleg K. Cheremnykh ${ }^{1}$, Volodymyr V. Koshovy ${ }^{3}$, Mykola O. Melnik ${ }^{4}$, Oleh L. Ivantyshyn ${ }^{3}$, \\ Roman T. Nogach ${ }^{4}$, Yuriy A. Selivanov ${ }^{1}$, Vladimir V. Grimalsky ${ }^{5}$, Valentyn P. Mezentsev ${ }^{4}$, Larysa M. Karataeva ${ }^{4}$, \\ Vasyl. M. Ivchenko ${ }^{2}$, Gennadi P. Milinevsky ${ }^{2}$, Viktor N. Fedun ${ }^{6}$, and Eugen N. Tkachenko ${ }^{2}$ \\ ${ }^{1}$ Space Research Institute NASU-SSAU, Kyiv, 03187, Ukraine \\ ${ }^{2}$ Taras Shevchenko National University of Kyiv, Kyiv, 01601, Ukraine \\ ${ }^{3}$ G. V. Karpenko Physical-Mechanical Institute NASU, Lviv, 79053, Ukraine \\ ${ }^{4}$ Lviv Centre of the Institute of Space Research NASU-NSAU, Lviv, 79053, Ukraine \\ ${ }^{5}$ Autonomous University of State Morelos (UAEM), Cuernavaca, Morelos, 62209, Mexico \\ ${ }^{6}$ Department of Automatic Control and Systems Engineering, The University of Sheffield, Sheffield, S1 3JD, UK
}

Correspondence to: Yuriy G. Rapoport (yuriy.rapoport@gmail.com)

Received: 16 August 2014 - Revised: 20 November 2016 - Accepted: 28 November 2016 - Published: 5 January 2017

\begin{abstract}
We develop theoretical basics of active experiments with two beams of acoustic waves, radiated by a ground-based sound generator. These beams are transformed into atmospheric acoustic gravity waves (AGWs), which have parameters that enable them to penetrate to the altitudes of the ionospheric $\mathrm{E}$ and $\mathrm{F}$ regions where they influence the electron concentration of the ionosphere. Acoustic waves are generated by the ground-based parametric sound generator (PSG) at the two close frequencies. The main idea of the experiment is to design the output parameters of the PSG to build a cascade scheme of nonlinear wave frequency downshift transformations to provide the necessary conditions for their vertical propagation and to enable penetration to ionospheric altitudes. The PSG generates sound waves (SWs) with frequencies $f_{1}=600$ and $f_{2}=625 \mathrm{~Hz}$ and large amplitudes $\left(100-420 \mathrm{~m} \mathrm{~s}^{-1}\right)$. Each of these waves is modulated with the frequency of $0.016 \mathrm{~Hz}$. The novelty of the proposed analytical-numerical model is due to simultaneous accounting for nonlinearity, diffraction, losses, and dispersion and inclusion of the two-stage transformation (1) of the initial acoustic waves to the acoustic wave with the difference frequency $\Delta f=f_{2}-f_{1}$ in the altitude ranges $0-0.1 \mathrm{~km}$, in the strongly nonlinear regime, and (2) of the acoustic wave with the difference frequency to atmospheric acoustic gravity waves with the modulational frequency in the altitude ranges $0.1-20 \mathrm{~km}$, which then reach the altitudes of the ionospheric $\mathrm{E}$ and $\mathrm{F}$ regions, in a practically lin-
\end{abstract}

ear regime. AGWs, nonlinearly transformed from the sound waves, launched by the two-frequency ground-based sound generator can increase the transparency of the ionosphere for the electromagnetic waves in $\mathrm{HF}(\mathrm{MHz})$ and VLF $(\mathrm{kHz})$ ranges. The developed theoretical model can be used for interpreting an active experiment that includes the PSG impact on the atmosphere-ionosphere system, measurements of electromagnetic and acoustic fields, study of the variations in ionospheric transparency for the radio emissions from galactic radio sources, optical measurements, and the impact on atmospheric aerosols. The proposed approach can be useful for better understanding the mechanism of the acoustic channel of seismo-ionospheric coupling.

Keywords. Electromagnetics (wave propagation)

\section{Introduction}

The generation of low-frequency sound waves in advance of earthquakes forms a part of the modern description of the processes in the upper crust. A paper by Xia et al. (2011) described 240 abnormal infrasound signals that have been observed in Beijing and, in total, 92 cases worldwide for earthquakes with $M \geq 7.0$ during 2002-2008. In this article, it is found that about $85 \%$ of the earthquakes are preceded by infrasound signals 1-30 days in advance, whilst earthquakes with $M \geq 7.6$ are most likely led by infrasound 1-10 days 
in advance. According to Xia et al. (2011), the frequency of co-seismic infrasound waves is roughly equal to $1 \mathrm{~Hz}$, while the frequency of waves observed before earthquakes is lower and in the $0-0.005 \mathrm{~Hz}$ frequency band.

During the last 25 years the presence of this phenomena occurring in both the ionosphere and atmosphere before earthquakes has been found. These phenomena are, in particular, variations in total electron content (TEC) (Liu et al., 2014), variations in the surface thermal anomalies (Genzano et al., 2007), increase and anomalous variability in outgoing long-wave radiation (Ouzounov et al., 2007), and the abnormal release of the heat energy (Kafatos et al., 2007). There are two main mechanisms of seismo-ionospheric phenomena, one of which is connected with electromagnetic and the other with atmosphere acoustic and internal gravity waves (Hayakawa, 2015; Pulinets and Boyarchuk, 2004). In addition, a mixed electromagnetic-atmospheric gravity wave mechanism may be important. In particular, atmosphere gravity waves can be excited due to the above-mentioned thermal anomalies. To realize the possibility of earthquake prediction, it is important to understand the mechanisms of seismo-ionospheric coupling, in particular, to distinguish the two possible mechanisms mentioned above. The present investigation proposes the mechanism of the penetration of atmospheric acoustic gravity waves from the lower atmosphere into the ionosphere. It is very useful to look, both theoretically and experimentally, how controllable sound from a ground-based generator would penetrate to the ionospheric altitudes and what effects they would cause in the ionosphere.

The acoustic lithosphere-ionosphere coupling is increasingly considered as one of the important factors of seismoionospheric phenomena (Sorokin and Hayakawa, 2013; Klimenko et al., 2011). The need for modelling of powerful disasters such as earthquakes (Gokhberg and Shalimov, 2000) has produced many studies of the acoustic effects in the ionosphere of powerful nuclear and technical explosions. In particular, the effect of the transformation of acoustic perturbations into electromagnetic waves was clearly demonstrated in the experiment MASSA (Gokhberg and Shalimov, 2000). However, the interpretation of the collection of natural and technical acoustic impacts on the ionosphere is quite diverse.

In particular, as shown in the paper Zettergren and Snively (2013), acoustic waves generated by tropospheric sources may attain significant amplitudes in the ionosphere, achieving temperature and vertical wind perturbations on the order of approximately tens of kelvins and metres per second throughout the $\mathrm{E}$ and $\mathrm{F}$ regions. The perturbations of the total electron content are predicted to be detectable by groundbased radar and GPS receivers. Acoustic waves also drive field-aligned currents that may be detectable by in situ magnetometers (Iyemori et al., 2015). A recent paper (Zettergren and Snively, 2015) reports that the recent measurements of GPS-derived TEC reveals acoustic wave periods from $\sim 1$ to $4 \mathrm{~min}$ in the F-region ionosphere following natural hazard events, such as earthquakes, severe weather fronts, and volcanoes.

Moreover, in order to search the seismogenic effects in the ionosphere, it is necessary to unify the well-developed model of the electromagnetic channel to seismo-ionospheric coupling (Molchanov et al., 1995; Rapoport et al., 2004b; Grimalsky et al., 1999; Pulinets and Boyarchuk, 2004; Sorokin and Hayakawa, 2013) with the model of the acoustic channel (Gokhberg and Shalimov, 2000; Rapoport et al., 2004a, 2009; Koshevaya et al., 2002; Grimalsky et al., 2003; Zettergren and Snively, 2015; Iyemori et al., 2015). Traditionally, AGWs and electromagnetic waves have been considered as the basis of two main competitive mechanisms of seismo-ionospheric coupling (Sorokin and Hayakawa, 2013; Klimenko et al., 2011; Pulinets and Boyarchuk, 2004). The synergy approach has been put forward recently (Pulinets, 2011). We are also working in the field of the unified description of electromagnetic and acoustic channels of seismoionospheric coupling (Rapoport et al., 2014a, b).

This research field is important in relation to natural hazards, being able to provide a new basis for the warning of the occurrence of devastating natural disasters such as volcanic eruptions and earthquakes. The subject of the current paper is the consideration of the acoustic channel in the interaction between the lower atmosphere and the ionosphere. The acoustic channel of seismo-ionospheric coupling can be investigated by means of active experiments on the penetration of modulated intense sound waves from man-made sources from the Earth's surface into the ionosphere. It is connected, in particular, with the role of the frequency transformation of sound excited by the parametric sound generator (PSG), including its experimental and theoretical investigation (Parrot et al., 2007; Koshovyy, 1999; Kotsarenko et al., 1999; Koshevaya et al., 2005).

In Koshevaya et al. (2005), the use of an acoustic generator is as the source of the acoustic signal that is similar to natural hazards caused by underground eruptions. This approach may help to investigate the acoustic signal effects in the ionosphere that should be released by the PSG (Soroka et al., 2006; Aramyan et al., 2008). In previous papers (Kotsarenko et al., 1999; Grimalsky et al., 2003; Koshevaya et al., 2005), the theory of the sound wave penetration produced by the two-frequency PSG has been proposed. Kotsarenko et al. (1999) described the change in the ionosphere transparency for radio waves from galactic radio sources that is connected with the spatial resonance between the periodical variations in the plasma density caused by AGWs and the radio waves. The influence of nonlinearity, gravity, dissipation (in the form of viscosity), non-isothermality, and diffraction on the infrasonic waves generated by the PSG has been considered (Koshevaya et al., 2005). Important theoretical results (Koshevaya et al., 2005) have demonstrated the nonlinear frequency conversion in the course of the penetration of intermediate frequency sound waves (ISWs) into the atmosphere with further transformation to AGWs and a change in 
transparency for radio waves, propagating through the nonuniform plasma due to nonlinear variation in the plasma density on average within the ionospheric F layer. In particular, this process is quite effective when the frequency of the radio wave is near the lower edge of the transparency band. The change in the infrasonic signal spectrum excited by the PSG, when it penetrates into the ionosphere, was considered a the recent paper by Krasnov and Kuleshov (2014).

In the present paper, we continue the experimental and theoretical works (Parrot et al., 2007; Koshovyy, 1999; Kotsarenko et al., 1999; Koshevaya et al., 2005; Grimalsky et al., 2003) on the penetration of SWs excited by the PSG into the atmosphere and ionosphere. We formulate and solve the new problems not considered in previous papers (Koshevaya et al., 2005; Krasnov and Kuleshov, 2014; Rapoport, et al., 2004). We propose a new hydrodynamic analyticalnumerical model which includes the two-stage (consequent) transformation of SWs: (1) parametric transformations of two SW packets with frequencies $\sim 600 \mathrm{~Hz}$ and a modulation frequency $\sim 0.01 \mathrm{~Hz}$ to ISWs with the carrier difference frequency in the ELF range $\sim(10-100) \mathrm{Hz}$ over a range of altitudes $(0-0.5) \mathrm{km}$, and (2) the transformation of ISWs in the ELF range with the carrier frequency $\sim(10-100) \mathrm{Hz}$ to AGWs in the ULF range with a frequency $\sim 0.01 \mathrm{~Hz}$. This model is based on the parameters of the experiments with the PSG and corresponds to the experimental results qualitatively well (Parrot et al., 2007; Koshovyy, 1999; Kotsarenko et al., 1999; Soroka et al., 2006; Aramyan et al., 2008). We then discuss the physical consequences of these processes and the possibilities of their observations using satellites and ground-based instruments.

We will present in this paper the analytical-numerical model of neutral atmospheric disturbances. Then, the results of the corresponding modelling will be used for the evaluations of some effects caused by AGWs, penetrating from the lower atmosphere into the ionospheric altitudes, on the ionospheric plasma and propagation of the electromagnetic waves in the ionosphere. The comparison with the experiment is connected with the analysis of the propagation time of acoustic gravity waves from the lower atmosphere to ionosphere altitudes as well as the dynamics of the neutral excitations, including their amplitudes and characteristic transverse sizes of the region of hydrodynamic excitations at ionospheric altitudes.

A possible impact of AGWs on the ionosphere has been estimated from the simulated amplitudes of the velocity of the neutral component of the ionosphere in AGWs at different heights and the evolution of transverse sizes of AGW beams. In addition, the experimental results on the increase in the ionosphere transmission/transparency for $\mathrm{HF}$ and VLF electromagnetic waves, obtained using the radio telescope URAN (Koshovy et al., 1997; Koshovyy and Soroka, 1998; Koshovyy, 1999; Kotsarenko et al., 1999) and satellite DEMETER (Parrot et al., 2007), are discussed. The increase in HF and VLF electromagnetic/radio wave intensity and ionospheric transparency for these waves are presented by Helliwell (1965) for ground-based measurements and in DEMETER (Parrot et al., 2007; Soroka et al., 2006) by satellite observation methods. We present simple evaluations for the effects in the ionosphere, and the detailed theory of the ionospheric response will be presented in subsequent papers.

Finally, the idea for a future active ground-satellite experiment involving electromagnetic and acoustics signals that are possibly connected with optical glows and effects in aerosol response (Aramyan et al., 2008) is formulated.

\section{The model of the double frequency transform}

The model includes the ground-based sound generator, which produces the strong acoustic emission with spectrum at the two SW frequencies $f_{1}=625 \mathrm{~Hz}$ and $f_{2}=600 \mathrm{~Hz}$. This acoustic emission is modulated by an ultra-low frequency (ULF) $F=\Omega / 2 \pi \sim 0.01 \mathrm{~Hz}$. As the SWs move upwards, due to the nonlinearity of the atmosphere, numerous harmonics of the SWs are excited, as well as ISWs at the difference frequency $f=f_{1}-f_{2}$. The sound wave harmonics dissipate at low heights $z<0.1 \mathrm{~km}$, whereas ISWs penetrate into the higher atmosphere and lower ionosphere, up to heights $z<100 \mathrm{~km}$. In turn, the ISWs produce harmonics jointly with the AGWs at the modulation frequency $F$. The harmonics of intermediate frequency sound waves dissipate in the upper atmosphere and lower ionosphere. AGWs can reach the $\mathrm{E}$ and $\mathrm{F}$ layers of the ionosphere (Koshevaya et al., 2005). The generation of the difference acoustic frequency $f$ can be referred to as the parametric frequency conversion, whereas the release of the modulation frequency $F$ can be considered as the acoustic detection. The sound generator used here is called the parametric sound generator, due to the generation of the difference frequency $f$. This double frequency transform of the SWs at the frequencies $f_{1,2}$ into ISWs at $f$ converted to AGWs at $F$ frequency is analysed below. An approximation of the moderate nonlinearity is used.

The nonlinear propagation of the acoustic pulses in the atmosphere is described by the following set of hydrodynamic equations (Rudenko, 1995):

$$
\begin{gathered}
\rho_{0}\left(\frac{\partial v_{z}}{\partial t}+v_{z} \frac{\partial v_{z}}{\partial z}\right)+\rho^{\prime} \frac{\partial v_{z}}{\partial t} \approx-\frac{\partial p^{\prime}}{\partial z}+\frac{\partial}{\partial z}\left(\rho_{0} v(z) \frac{\partial v_{z}}{\partial z}\right)-\rho^{\prime} g ; \quad(1) \\
\rho_{0} \frac{\partial \boldsymbol{v}_{\perp}}{\partial t} \approx-\nabla_{\perp} p^{\prime} ; \frac{\partial \rho^{\prime}}{\partial t}+\frac{\partial}{\partial z}\left(\rho_{0} v_{z}\right)+\rho_{0} \operatorname{div} \boldsymbol{v}_{\perp}+\frac{\partial}{\partial z}\left(\rho^{\prime} v_{z}\right) \approx 0 ; \\
\frac{\partial p^{\prime}}{\partial t}-\rho_{0} g v_{z}+v_{z} \frac{\partial p^{\prime}}{\partial z} \approx-c_{\mathrm{s}}^{2}\left(\rho_{0} \frac{\partial v_{z}}{\partial z}+\rho_{0} \operatorname{div} \boldsymbol{v}_{\perp}+\gamma \rho^{\prime} \frac{\partial v_{z}}{\partial z}\right) .
\end{gathered}
$$

Here $\left(v_{x}, v_{y}, v_{z}\right)$ are the components of the air velocity $\left(\boldsymbol{v}_{\perp} \equiv\left(v_{x}, v_{y}\right)\right), \rho$ and $p$ are its total density and pressure, and $g$ is the free-fall acceleration. We write $\rho=\rho_{0}+\rho^{\prime}$, $p=p_{0}+p^{\prime}$, where $\rho_{0}$ and $p_{0}$ are the stationary values of the atmospheric density and pressure and $\rho^{\prime}$ and $p^{\prime}$ are the nonstationary parts. Also, the adiabatic equation for pressure 

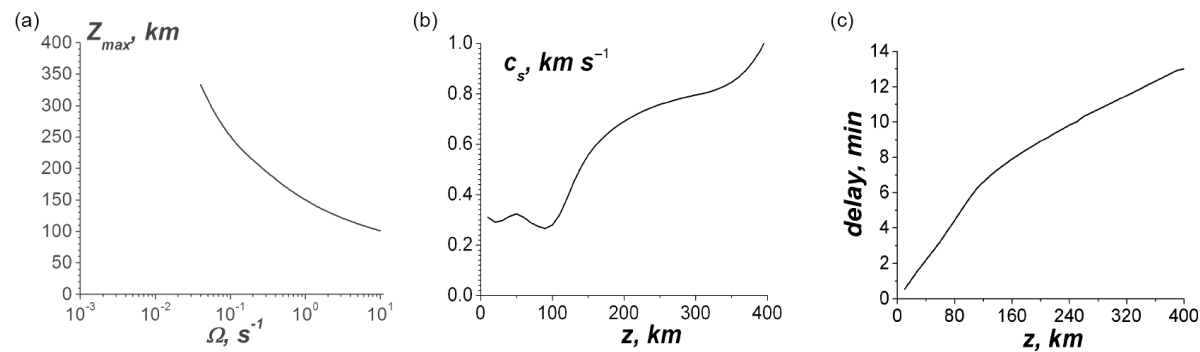

Figure 1. (a) The dependence of the heights $Z_{\max }$ on the circular frequency $\Omega \equiv 2 \pi F$ of ULF AGWs, where the maximum values of the air velocity in ULF AGWs occur; (b) the dependence of the sound velocity $c_{\mathrm{S}}$ on the height; and (c) the dependence of the propagation time $\int_{0}^{z} \frac{\mathrm{d} z}{c_{\mathrm{s}}(z)}$, or time delay, of acoustic waves on the height.

is used with the adiabatic constant $\gamma$. The kinematic viscosity of air is $v(z)$, which increases drastically with the height $z$. The presence of thermoconductivity, or non-adiabaticity, in the equation for the pressure results in an increase in the dissipation of about $20 \%$. A moderate nonlinearity is assumed, so that only quadratic nonlinear terms are preserved in Eq. (1). Also, it is assumed that the acoustic wave moves preferentially vertically upwards and inequalities $\left|v_{x, y}\right|<v_{z}$ are valid.

To consider the propagation of acoustic waves in the atmosphere and the ionosphere, it is necessary to take into account the dependencies of kinetic temperature $T$, molar mass $M$ and effective atmosphere scale height $H$ on the vertical coordinate $z$. Generally, the effective atmosphere height is not a constant. The dependencies of the density, sound velocity, and viscosity on height $z$ are (Jursa, 1985)

$$
\begin{gathered}
\frac{\mathrm{d} \rho_{0}(z)}{\mathrm{d} z}=-\frac{\rho_{0}(z)}{H(z)} ; c_{\mathrm{S}}(z)=c_{\mathrm{S}}(z=0)\left(\frac{T(z)}{T(z=0)} \frac{M(z=0)}{M(z)}\right)^{1 / 2} ; \\
v(z)=v(z=0)\left(\frac{T(z)}{T(z=0)}\right)^{1 / 2} \frac{1+\frac{S}{T(z=0)}}{1+\frac{S}{T(z)}} w^{2}
\end{gathered}
$$

Here the following function $w(z)$ is used:

$w(z)=\exp \left(\int_{0}^{z} \frac{\mathrm{d} z^{\prime}}{2 H\left(z^{\prime}\right)}\right), \frac{\mathrm{d} w(z)}{\mathrm{d} z}=\frac{w(z)}{2 H(z)}$.

$S$ is the Sutherland constant $(S=110.4 \mathrm{~K})$. Note that dependencies of $H(z), T(z)$, and $M(z)$ possess a characteristic scale of about $20-30 \mathrm{~km}$, so that it is possible to neglect their derivatives with respect to $z$.

The dependencies $T(z), H(z), M(z)$, and $c_{\mathrm{S}}(z)$ are taken from Jursa (1985). Note that, at heights $z>130 \mathrm{~km}$, an essential growth of the sound velocity takes place. The dependencies of the temperature and the molar content of the ionosphere are very important for the propagation of AGWs; see Fig. 1b. The velocities of AGWs at the frequencies $F>$ $0.002 \mathrm{~Hz}$ and SWs at the frequencies $f>1 \mathrm{~Hz}$ are practically the same, i.e. differ within $10 \%$. Propagation of AGWs to the altitudes higher than $Z=400 \mathrm{~km}$ is not considered here, because only AGWs at the frequencies $F<10^{-3} \mathrm{~Hz}$ could penetrate to these heights (see Fig. 1a) where the hydrodynamic model is doubtful. The maximum heights of the wave propagation have been calculated within an approximation of small amplitudes of plane waves without nonlinearity. Moreover, an influence of AGWs on the propagation of electromagnetic waves in the $\mathrm{kHz}$ and $\mathrm{MHz}$ wave bands, where the AGW influence takes place within $\mathrm{E}$ and $\mathrm{F}$ layers at the lower heights $Z<400 \mathrm{~km}$, is considered in this paper.

Due to the wave acoustic dispersion being small for the circular frequencies $\geq 0.1 \mathrm{~s}^{-1}$, many harmonics $\geq 10$ are excited under the nonlinear interaction. Therefore, a slowly varying profile approach is suitable for the analysis of the nonlinear acoustic propagation in the atmosphere. It is possible to derive a single equation for the vertical component of the velocity of air $v_{z}$, where a dependence on only one transverse cylindrical coordinate $\rho$ is assumed, $\partial / \partial \varphi=0$. When introducing a new dependent variable $V=v_{z} / w$, and the independent ones $z, \eta=t-\int_{0}^{z} \frac{\mathrm{d} z^{\prime}}{c_{\mathrm{s}}\left(z^{\prime}\right)}, \rho$, the resulting equation is

$$
\begin{aligned}
\frac{\partial}{\partial \eta}\left(\frac{\partial V}{\partial z}\right. & \left.-\frac{v(z)}{2 c_{\mathrm{s}}(z)^{3}} \frac{\partial^{2} V}{\partial \eta^{2}}-\frac{1+\gamma}{2} \frac{w(z)}{2 c_{\mathrm{s}}(z)^{2}} \frac{\partial}{\partial \eta}\left(V^{2}\right)\right) \\
& +\frac{c_{\mathrm{s}}(z)}{2 H(z)^{2}} V=\frac{c_{\mathrm{s}}}{2} \Delta_{\perp} V .
\end{aligned}
$$

Equation (4) is the Khokhlov-Zabolotskaya equation (Rudenko, 1995) in the case of a medium with the density decreasing along the $z$ axis. Here, we consider the case where the lowest modulation frequency is $\Omega \sim 0.05-1 \mathrm{~s}^{-1}>\Omega_{\mathrm{a}} \equiv$ $c_{\mathrm{s}} / 2 H \approx 0.02 \mathrm{~s}^{-1}$, where subscript "a" stands for the acoustic cut-off frequency. After changing the independent variable from $z$ to $w(\mathrm{~d} z / \mathrm{d} w=2 H(z) / w)$, it is possible to rewrite Eq. (4) as

$$
\begin{aligned}
\frac{\partial}{\partial \eta}\left(\frac{\partial V}{\partial w}\right. & \left.-\frac{\nu(0) H w}{c_{\mathrm{S}}(z)^{3}} \frac{1+\frac{S}{T(0)}}{1+\frac{S}{T(z)}} \frac{\partial^{2} V}{\partial \eta^{2}}-\frac{1+\gamma}{2} \frac{H(z)}{c_{\mathrm{S}}(z)^{2}} \frac{\partial}{\partial \eta}\left(V^{2}\right)\right) \\
& +\frac{c_{\mathrm{S}}(z)}{4 H(z) w} V=\frac{c_{\mathrm{S}}(z) H(z)}{w} \Delta_{\perp} V .
\end{aligned}
$$

The consequent frequency (and spectrum) transform under vertical propagation of acoustic beams is investigated. At 
the Earth's surface the sound generator excites the relatively powerful SWs at two (carrier) frequencies $f_{1}, f_{2}\left(\omega_{1,2}=\right.$ $\left.2 \pi f_{1,2}\right)$.

\subsection{The first frequency transform}

This transform is as follows: the initial (carrier) SW frequencies are $f_{1}=625 \mathrm{~Hz}, f_{2}=600 \mathrm{~Hz}$, or, for the sake of comparison, $f_{2}=525 \mathrm{~Hz}$, where the difference frequency is 4 times greater. This transform takes place in the first altitude region $0 \leq z \leq z_{1}$, where its upper boundary is $z_{1}$ $\approx 0.05 \mathrm{~km}$. The amplitudes of the air velocity at $z=0$ are $v(z=0) \equiv v_{0}=(100-420) \mathrm{m} \mathrm{s}^{-1}$ and the boundary condition at the Earth's surface $z=0$ (or $w=1$ ) is

$$
\begin{aligned}
V(w & =1, t, \rho)=\frac{v_{0}}{2}\left(\cos \left(\omega_{1} t\right)+\cos \left(\omega_{2} t\right)\right) \cdot \exp \left(-\left(\rho / \rho_{01}\right)^{2}\right) \\
& \equiv v_{0} \cos \left(\omega_{0} t\right) \cos \left(\frac{\omega_{1}-\omega_{2}}{2} t\right) \cdot \exp \left(-\left(\rho / \rho_{01}\right)^{2}\right)
\end{aligned}
$$

The maximum experimental value of the amplitude of the input velocity is $v_{0}=420 \mathrm{~m} \mathrm{~s}^{-1}$ for our setup (Aramyan et al., 2008). The initial width of the SW beam is $\rho_{01}=(1-10) \mathrm{m}$ in the horizontal plane. For the experimental setup, it is $\rho_{01} \approx$ $1 \mathrm{~m}$. The carrier circular frequency is $\omega_{0}=\left(\omega_{1}+\omega_{2}\right) / 2$. The first frequency transform is the nonlinear generation of the difference frequency $f$, or intermediate circular frequency $\omega_{1}-\omega_{2}: f_{0} \rightarrow f=\left(\omega_{1}-\omega_{2}\right) / 2 \pi$. It is supposed that $t \ll$ $\Omega^{-1}$ and the value $\Omega$ is not included into the formulas of this section. Equation (5) is solved by the spectral method when the following expression is used:

$$
\begin{aligned}
V(w, \eta, \rho) & =\frac{1}{2} \sum_{n=1,2, \ldots} A_{n}(w, \eta, \rho) \exp \left(\text { in } \omega_{0} \eta\right) \\
& +(\text { c.c. })+A_{0}(w, \eta, \rho) .
\end{aligned}
$$

Here $A_{n}, n=1,2, \ldots$ are the slowly varying amplitudes of the carrier frequency harmonics and $A_{0}$ is the lower frequency part with the spectrum near the intermediate frequency $f$. For $A_{n}$ and $A_{0}$, the following set of coupled equations has been obtained:

$$
\begin{aligned}
& \frac{\partial A_{n}}{\partial w}+ \Gamma w\left(n \omega_{0}\right)^{2} A_{n}-i n \omega_{0} N \\
& \cdot\left(\frac{1}{2} \sum_{p<n} A_{p} A_{n-p}+\sum_{p>n} A_{p} A_{n-p}^{*}\right)=0 \\
& \frac{\partial}{\partial \eta}\left(\frac{\partial A_{0}}{\partial w}-\Gamma w \frac{\partial^{2} A_{0}}{\partial \eta^{2}}-\frac{N}{2} \frac{\partial}{\partial \eta} \sum_{p}\left|A_{p}\right|^{2}\right)+\frac{\alpha}{w} A_{0} \\
&=\frac{D}{w} \Delta_{\perp} A_{0} .
\end{aligned}
$$

The boundary conditions for the harmonics are

$A_{1}(w=1)=v_{0} \cos (\omega t) \cdot \exp \left(-\left(\rho / \rho_{01}\right)^{2}\right)$,

$A_{2,3 \ldots}(w=1)=0, A_{0}(w=1)=0$.

Here $\Gamma, \alpha, N$, and $D$ are expressed through the parameters of the atmosphere used in Eq. (5). The wave diffraction is es- sential for the wave at lower frequencies $A_{0}$. A lot of higher harmonics $(\geq 20)$ of the SWs are generated, but they dissipate at small vertical distances $z<0.02 \mathrm{~km}$.

As a result of the nonlinear interaction of the SWs frequency components $f_{1,2}$, the ELF ISW at the difference, or intermediate, frequency $f=f_{1}-f_{2}=25-100 \mathrm{~Hz}$ is generated at the vertical distances $z=0.01-0.05 \mathrm{~km}$. Its velocity amplitude is $v_{0}=2-20 \mathrm{~m} \mathrm{~s}^{-1} \sim v_{1}\left(f / f_{1}\right)$ (see Eq. 6) and the transverse width of the beam is $\rho_{02}=50-200 \mathrm{~m}$. Note that the frequency $F$ is the modulation frequency also for the ISW. This ISW propagates vertically upwards over the distances $z \leq 20 \mathrm{~km}$. The second frequency transform occurs in the ranges of altitudes from $100 \mathrm{~m}$ to $20 \mathrm{~km}$. The higher harmonics are generated from the ISW at the intermediate frequency jointly with the generation of AGWs at the modulation frequency $F=\Omega / 2 \pi$. Thus, the second frequency transform $f \rightarrow F$ takes place in the ranges of altitudes from dozens of metres to $20 \mathrm{~km}$.

\subsection{The second frequency transform}

The second frequency transform is described by the set of equations with the same form as Eqs. (7) and (8), but the acoustic harmonics are of the intermediate frequency generated under the first frequency transform and the lower frequency component is AGWs. The difference between the first frequency transform and the second one is in the spatial scales and the values of air velocities within the acoustic wave.

Equation (5) is augmented by the boundary conditions to simulate this second frequency transform:

$$
\begin{gathered}
V\left(w=w_{1}, t, \rho\right)=V_{0} \cos (\omega t) \cdot \cos \left(\frac{\Omega}{2} t\right) \cdot \Phi(\rho) ; \\
\Phi(\rho) \approx \exp \left(-\left(\rho / \rho_{02}\right)^{2}\right) ; \Phi\left(\rho_{02}\right)=0.5 \Phi(0) .
\end{gathered}
$$

Here $V\left(w=w_{1}, t, \rho\right)$ is the velocity of the ISW at the lower boundary $z=z_{1}$ of the second altitude region $z \geq z_{1}$. The initial transverse profile $\Phi(\rho)$ and the amplitude $V_{0}$ has been taken in Eq. (9) as the profile of the ISW, i.e. at the intermediate frequency $f$ generated in the first frequency transform at the upper boundary $z_{1} \approx 0.05 \mathrm{~km}\left(w_{1}=\exp \left(z_{1} / 2 H\right)\right)$ of the first altitude region $0 \leq z \leq z_{1}$, where SWs at higher frequencies $f_{1,2}$ completely dissipate. The second equation in the second line from Eq. (9) is, in fact, the determination of the value $\rho_{02}$. The amplitude of ISW $V_{0}$ at $z=z_{1}$ is equal to the maximum value of $A_{0}$ computed for the conversion of SWs to ISW from the second of Eq. (8) in the centre $\rho=0$ : $V_{0}=\left(A_{0}\left(z=z_{1}, \rho=0, t\right)\right)_{\max }$. Thus, the equations

$$
\begin{aligned}
& A_{1}\left(w=w_{1}\right)=V_{0} \cos (\Omega t / 2) \cdot \Phi(\rho), A_{2,3 \ldots}\left(w=w_{1}\right)=0, \\
& A_{0}\left(w=w_{1}\right)=0
\end{aligned}
$$

are the boundary conditions for the harmonics for the second frequency transform. 
(a)

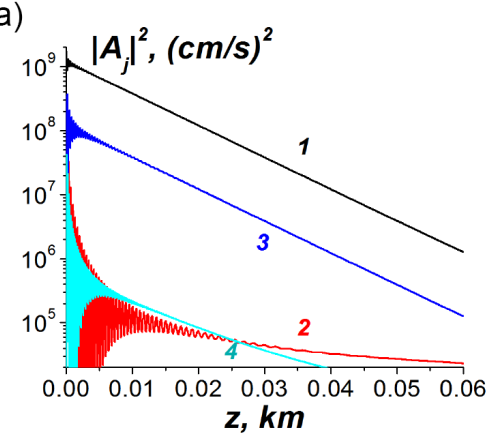

(c)

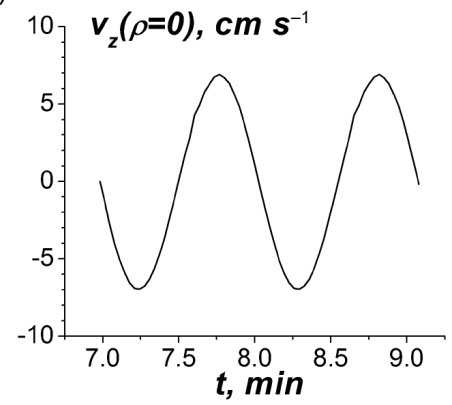

(b)

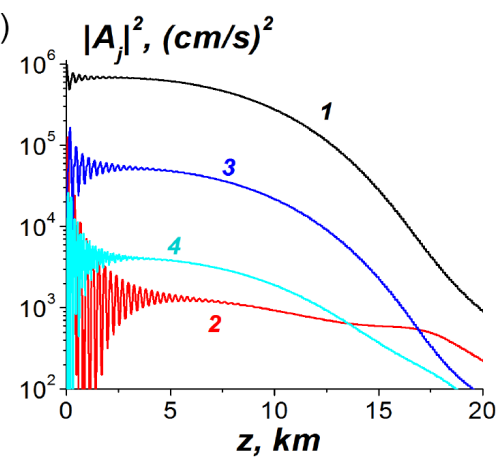

(d)

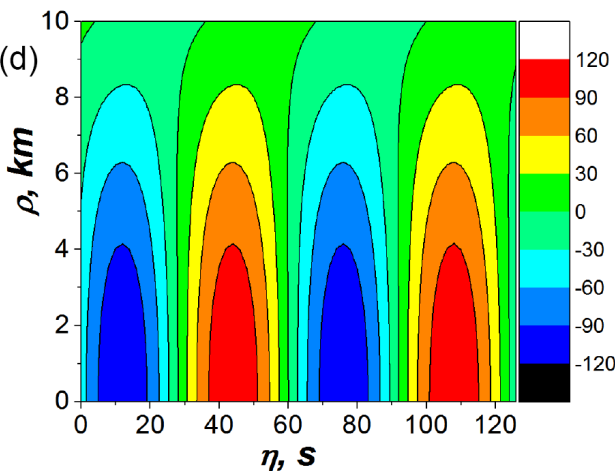

Figure 2. The double frequency transform $600 \mathrm{~Hz} \rightarrow 25 \mathrm{~Hz} \rightarrow 0.016 \mathrm{~Hz}$ from an initial transverse SW width of $1 \mathrm{~m}$ at a frequency of $600 \mathrm{~Hz}$ and an amplitude of $420 \mathrm{~m} \mathrm{~s}^{-1}$ : (a) the dependence of $\left|A_{j}\right|^{2}\left(A_{1}, A_{2}\right.$, etc. are maximum amplitudes of harmonics at the frequency $\left.600 \mathrm{~Hz}\right)$, (b) the dependence of $\left|A_{j}\right|^{2}$ (squares of the maximum amplitudes of harmonics of the intermediate frequency $f=25 \mathrm{~Hz}$ ) for the second transform, and (c, d) distributions of the air velocity $v_{z}$ in ULF AGWs ( $\mathrm{cm} \mathrm{s}^{-1}$ units) at the heights $z=130 \mathrm{~km}$ and $z=300 \mathrm{~km}$, respectively. The dependence $v_{z}(t)$ in the centre of the beam in panel (c) is given with an account of the time delay.

\subsection{Model of AGW penetration through the neutral atmosphere to the ionosphere}

During sound wave propagation, an intermediate wave frequency ISW at the intermediate frequency $f \sim 25 \mathrm{~Hz}$ produces $\geq 5$ harmonics. Also, due to ISW modulation by the ULF frequency $F \equiv \Omega / 2 \pi, \Omega=0.03-0.5 \mathrm{~s}^{-1}$, the ULF AGW is generated as a detection of the modulated signal at the circular frequency $\omega=2 \pi f$. The ULF part of the spectrum is not subject to dissipation; on the contrary, it increases due to this nonlinear interaction. When the transverse scale is $\rho_{02} \geq 0.2 \mathrm{~km}$ in Eq. (9), the diffraction is not important for the ISW at the difference frequency, $f$, but the diffraction can decrease the peak amplitude of the ULF AGW. The ULF AGWs can propagate upwards to heights $z=200-400 \mathrm{~km}$, depending on their frequencies. The maximum heights are $z=200 \mathrm{~km}$ for $\Omega=0.5 \mathrm{~s}^{-1}$ and $z=400 \mathrm{~km}$ for $\Omega=0.03 \mathrm{~s}^{-1}$. The amplitudes of ULF AGWs at the heights $z=10-20 \mathrm{~km}$ can reach the values $v=0.01-$ $0.3 \mathrm{~cm} \mathrm{~s}^{-1}$. Because of the decreasing air density, the maximum values of amplitudes of the velocity can reach the values $v=1-10 \mathrm{~m} \mathrm{~s}^{-1}$. In the ideal case, they can reach values of $v=100 \mathrm{~m} \mathrm{~s}^{-1}$, but it is not realistic for the parametric sound generator used here. It is interesting that the higher harmonics, in practice, are not excited for ULF AGWs, even for the maximum values $v=100 \mathrm{~m} \mathrm{~s}^{-1}$ at the heights $z \sim(250-300) \mathrm{km}$, due to the wave dispersion of AGWs. The final width of ULF AGWs is of about $1-5 \mathrm{~km}$ in the horizontal plane, and the wave diffraction is very important for the propagation of ULF AGWs.

As a result of the first frequency transform, the ISW at the intermediate frequency $f=f_{1}-f_{2}=25-100 \mathrm{~Hz}$ is generated at the vertical distances $z=0.02-0.05 \mathrm{~km}$. Its amplitude of the velocity is $v \equiv v_{0}=2-10 \mathrm{~m} \mathrm{~s}^{-1} \sim v_{0}\left(f / f_{1}\right)$, the width of the beam is $\rho_{02}=20-100 \mathrm{~m}$ in the horizontal plane. The ISW propagates vertically upwards up to distances $z=1-20 \mathrm{~km}$. As mentioned above, to investigate this frequency transform, Eq. (5) is augmented by the boundary conditions (Eq. 9) to simulate this transformation. In Eq. (9) the boundary condition is taken at $w_{1} \approx 1$, because the release of the intermediate frequency $f$ occurs at low heights $z<100 \mathrm{~m}$.

Selected results of simulations of the double frequency transform, or the double frequency down-conversion, are presented in Figs. 2 and 3. One can see that the air velocities within ULF AGWs can reach the values of $v \sim 10 \mathrm{~cm} \mathrm{~s}^{-1}$ at the heights $z=130 \mathrm{~km}$ in the ionospheric $\mathrm{E}$ layer and 

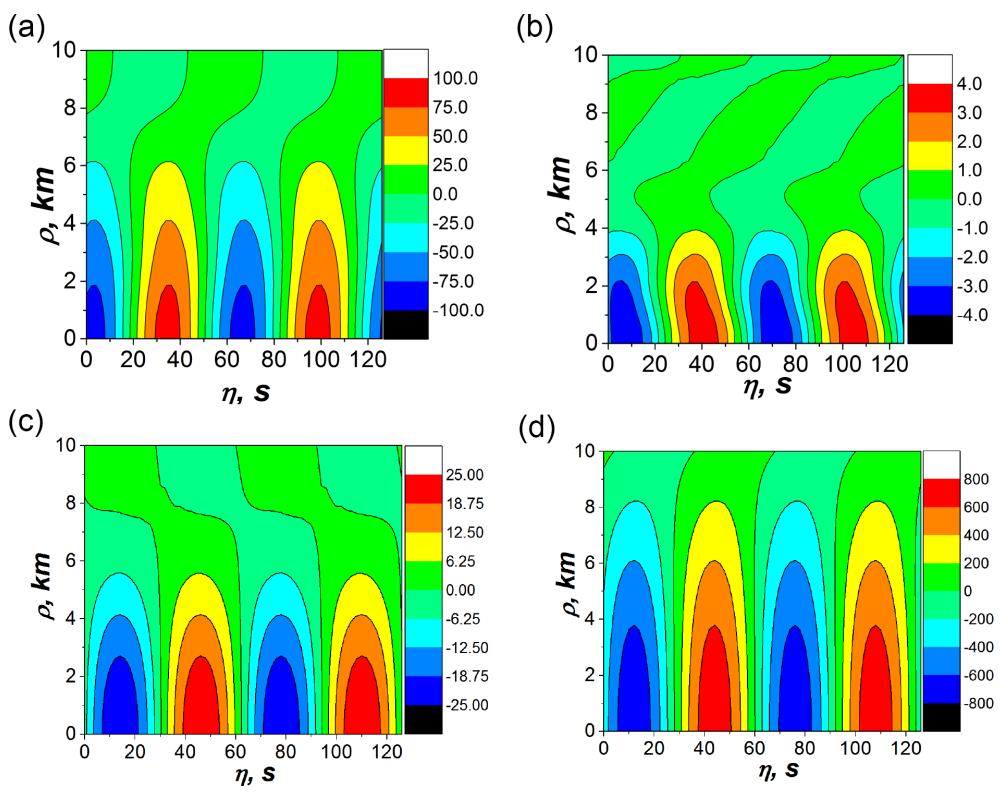

Figure 3. Spatial distribution of the air velocity $v$ in ULF AGWs ( $\mathrm{cm} \mathrm{s}^{-1}$ units) for different parameters. Panels (a) and (d) are for the double frequency transforms $600 \mathrm{~Hz} \rightarrow 25 \mathrm{~Hz} \rightarrow 0.016 \mathrm{~Hz}$, panel (c) is for $600 \mathrm{~Hz} \rightarrow 100 \mathrm{~Hz} \rightarrow 0.016 \mathrm{~Hz}$, and panel (b) is for the single frequency transform $25 \mathrm{~Hz} \rightarrow 0.016 \mathrm{~Hz}$. Panels (a), (b), and (c) are for an initial transverse SW width of $1 \mathrm{~m}$. Panel (d) is for an initial transverse SW amplitude of $2.5 \mathrm{~m}$. Panel (a) is for an initial transverse SW amplitude of $320 \mathrm{~m} \mathrm{~s}^{-1}$; and panels (b), (c), and (d) are for an initial transverse SW amplitude $420 \mathrm{~m} \mathrm{~s}^{-1}$. Panels (a) and (d) are for an altitude of $Z=300 \mathrm{~km}$; panels (b) and (c) are for an altitude of $Z=130 \mathrm{~km}$.

the values of more than $1 \mathrm{~m} \mathrm{~s}^{-1}$ at $z=300 \mathrm{~km}$ in the ionospheric $\mathrm{F}$ layer, when the intermediate frequency is $f=25 \mathrm{~Hz}$ (Fig. 2). In the case of the intermediate frequency $f=100 \mathrm{~Hz}$, the air velocities in ULF AGWs are 5 times greater (compare maximum values of $v$ in Fig. 3c with Fig. 2c). This can be explained by the preferential diffraction of ISW at lower frequencies. When the intermediate frequency $f$ is higher, more efficient conversion of SWs to ISW takes place, even neglecting diffraction, despite the greater dissipation at higher frequencies. The use of wider SW beams can reduce the influence of diffraction at lower frequencies (see Fig. 3d), where the initial SW beam is 2.5 times wider compared with Fig. 2d.

As our simulations have demonstrated, the amplitudes of the air velocity of ULF AGWs at $z \geq 100 \mathrm{~km}$ depend on the square of the input amplitude of SW until the values of the input air velocities $v_{0} \approx 300 \mathrm{~m} \mathrm{~s}^{-1}$. At the higher values of $v_{0}$, the saturation of the increase occurs. The result of the simulations of the double frequency transform for $z=300 \mathrm{~km}$ is given in Fig. 3a for the input amplitude $v_{0}=320 \mathrm{~m} \mathrm{~s}^{-1}$. The values of the air velocity are practically the same at the heights $z=130 \mathrm{~km}$ and $z=300 \mathrm{~km}$ (Fig. 3a), as for the input amplitude $v_{0}=420 \mathrm{~m} \mathrm{~s}^{-1}$ (see Fig. $2 \mathrm{~d}$ for $z=300 \mathrm{~km}$ ). Practically the same efficiency can be explained by the strong dissipation of SWs due to intense excitation of higher harmonics for the input amplitude $v_{0}=420 \mathrm{~m} \mathrm{~s}^{-1}$ (see Fig. 2a, b). At the input velocities $v_{0} \geq 340 \mathrm{~m} \mathrm{~s}^{-1}$, the non-adiabaticity of the motion of the air can be important, leading to ad- ditional sound dissipation. Thus, the difference of the efficiency of the frequency conversion for $v_{0}=320 \mathrm{~m} \mathrm{~s}^{-1}$ and $v_{0}=420 \mathrm{~m} \mathrm{~s}^{-1}$ can be even smaller.

The results of simulations of the one-stage frequency transform $25 \mathrm{~Hz} \rightarrow 0.016 \mathrm{~Hz}$ are given in Fig. $3 b$. The input amplitude at the frequency $25 \mathrm{~Hz}$ is $V_{0}=420 \mathrm{~m} \mathrm{~s}^{-1}$, the initial width is $1 \mathrm{~m}$, as for the double frequency transform in Fig. 2. The efficiency of the excitation of ULF AGWs at $z=130 \mathrm{~km}$ is twice lower than for the double frequency transform (compare maximum values shown in Fig. $3 \mathrm{~b}$ and Fig. 2c), due to the very strong diffraction of the input sound beam.

Our results have been compared with earlier simulations by Krasnov and Kuleshov (2014), where the input values of the air velocities were $\sim 10^{3}$ times smaller and the spectrum of ULF AGWs was wider. The one-stage frequency transformation was considered there. There is an agreement for the air velocities at the heights $z=130$ and $300 \mathrm{~km}$ for the corresponding ULF AGW components $F=0.01-0.03 \mathrm{~Hz}$. However, our simulations correspond to a different physical situation, i.e. an excitation of SWs by bounded man-made generators with a two-stage frequency transformation. The nonlinear generation of harmonics of powerful SWs considered in the present paper (Fig. 2a, c) is the essential mechanism of the dissipation. That is, when the ISW frequency is $f=25 \mathrm{~Hz}$, the nonlinear transform occurs at the heights $z \leq 20 \mathrm{~km}$ (see Fig. 2a, b), whereas in the purely linear case 
(a)

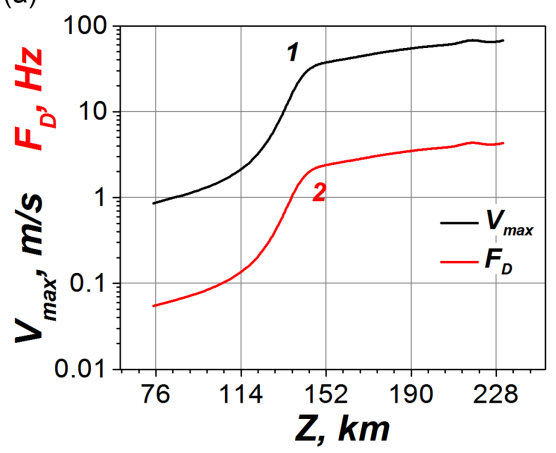

(b)

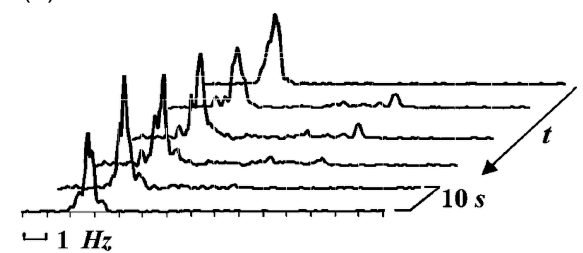

A 1

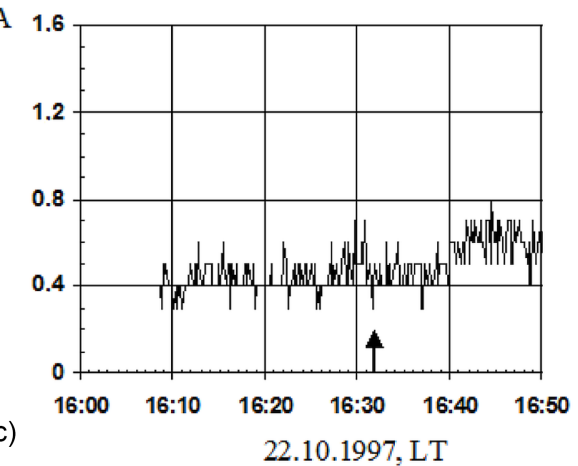

Figure 4. Panels (a) and (b) are experimental results on the measurements of the signal scattered from ionospheric inhomogeneities. Panel (a) shows the time dependences of the envelope of the scattered signal. The beginning of the acoustic irradiation is marked by an arrow. Panel (b) shows the spectra of the power of the scattered signal and (c) the simulation results. $V_{\max }$ is the maximum velocity of the air in ULF acoustic waves (curve 1) and $F_{\mathrm{D}}$ is the Doppler shift due to ULF acoustic wave (curve 2).

ISWs at this frequency can reach heights of $z=60-80 \mathrm{~km}$ (Rapoport et al., 2004).

\section{Experimental results}

In this section, we present the experimental results of the PSG-injected sound wave influence on the effective ionospheric radio wave transparency for the different frequency ranges measured by ground-based and satellite instruments.

\subsection{Artificial acousto-ionospheric disturbances investigation by URAN-3 radio telescope}

Experimental investigations with the use of a radio telescope can be realized both with ground-based sources and with the radio-astronomical method. The first one is the method of the scattering of HF radio waves by small-scale inhomogeneities of the electron concentration within the ionosphere. This method uses remote radar as $\mathrm{HF}(\mathrm{MHz})$ transmitter and the radio telescope URAN-3 as a receiver. The set of experiments were conducted in October 1997 (Koshovy and Ivantyshyn, 1998, 1999; Koshovyy, 1999). The signal frequency was $f_{\text {radio }}=16.173 \mathrm{MHz}$ and the incidence angle to the ionospheric inhomogeneity was $\theta_{\mathrm{I}}=38.6^{\circ}$. Ionospheric signals were registered with two polarizations at the intermediate frequency $3 \mathrm{~Hz}$ with the discretization frequency $35 \mathrm{~Hz}$. Typical realizations of the envelope of the signal $A(t)$ are presented in Fig. 4a. The averaging time was $10 \mathrm{~s}$. The beginning of the acoustic irradiation is marked by an arrow. Within the signal, it is possible to observe several characteristic regions. The first one is related to the non-perturbed ionosphere, whereas other ones can be related to the ionosphere modified by ULF acoustic waves. In the record from 22 December 1997, the magnitude of the signal is small with a low dispersion from the beginning until 16:38 LT, approximately $7 \mathrm{~min}$ after the beginning of the acoustic sounding. During the next time interval 7-13 min, until 16:50 LT, some increase in the magnitude of the scattered signal was observed, as well as the growth of the dispersion and lower frequency modulation of the envelope. A spectral analysis of the scattered ionospheric signal was made using a time window of $30 \mathrm{~s}$ with a sequential shift of $10 \mathrm{~s}$. This time window was of about half of the duration of the acoustic sounding. The results of the spectral analysis of six experimental results are presented in Fig. 4b. The Doppler frequency shift appeared at 16:37:30 LT, whereas the beginning of the acoustic sounding was 16:31:07 LT. We emphasize that the time difference is equal to $6 \mathrm{~min} 23 \mathrm{~s}$, which corresponds to the propagation time from the ground to the altitude $Z=160 \mathrm{~km}$ (see Fig. 1). Doppler shifts within the frequency interval 1$7 \mathrm{~Hz}$ were recorded during the experiment. Due to the signal modulation by frequency of $3 \mathrm{~Hz}$, the true Doppler frequency shift is the difference between the value in Fig. $4 \mathrm{~b}$ and this modulation frequency $(3 \mathrm{~Hz})$.

Experimental investigations of artificial acoustoionospheric disturbances were also conducted using the broadband registration system of the radio telescope URAN3 , which records the dynamic spectra of HF range, or the decametre wave range of the radio emission from galactic radio sources.

The acoustic wave injected by the controlled ground-based acoustic generator, which functioned in a single mode, consists of a sequence of intervals of the acoustic soundings with duration of $60 \mathrm{~s}$, each with a $60 \mathrm{~s}$ pause between them. 
(a)
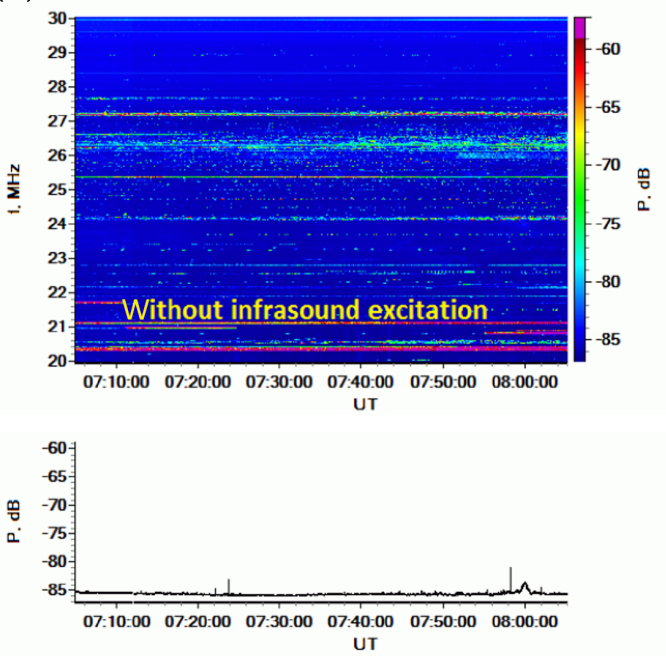

(b)
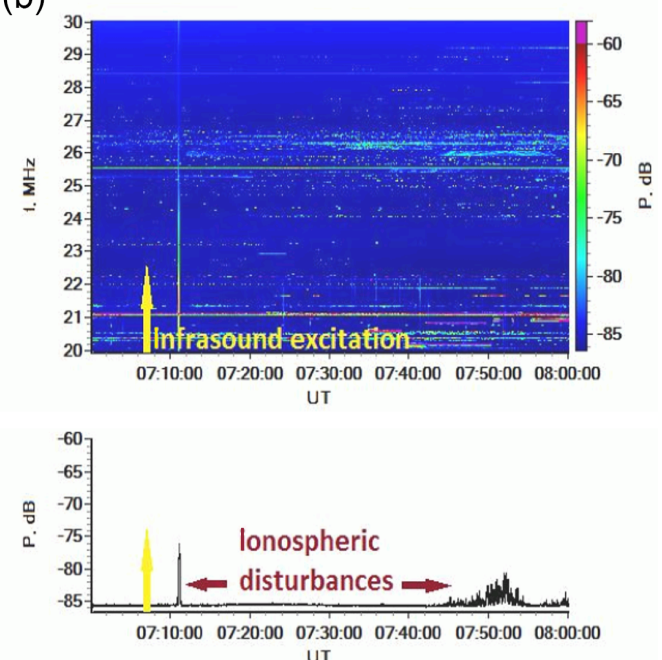

UT

Figure 5. (a) The galactic background radio emission without infrasound excitation has been observed with the radio spectrograph of the HF radio telescope URAN-3: dynamic spectrum (upper panel) and time profile at a frequency of $24 \mathrm{MHz}$ (lower panel). The galactic background radio emission in the absence of infrasound excitation was characterized by a fairly uniform level of background radio emission across the band of 20-30 MHz. (b) The galactic background radio emission with the infrasound excitation has been observed with the radio spectrograph of the HF radio telescope URAN-3: dynamic spectrum (upper panel) and time profile at a frequency of $24 \mathrm{MHz}$ (lower panel). After $\sim 4.5 \mathrm{~min}$ from the start of the infrasound excitation (yellow arrows) an increase in intensity of the galactic radio background has been observed.

The results of observations of the galactic background radio emission in the absence of infrasound excitation of the ionosphere (see Fig. 5a) and during the excitation (see Fig. 5b) are presented below. Measurements of the galactic background radio emission were carried out in a selected area of the celestial sphere, which was chosen to account for the locations of the radio telescope URAN-3 and the acoustic generator, as well as the estimated height that would be achieved by the waves.

Figure 5a shows the experimental record of the dynamic radio spectrum of galactic background in the band 20$30 \mathrm{MHz}$ (the upper part in Fig. 5a) and its time profile at a frequency of $24 \mathrm{MHz}$ (the lower part in Fig. 5a). These measurements were carried out on 24 November 2013 in the absence of infrasound excitation. Figure 5b shows the similar data record from 28 November 2013 in the period from 07:00:00 to 08:00:00 UT with infrasound excitation. The start time of the infrasound excitation (07:07:00 UT) is represented by the yellow arrow. The acoustic generator was operated in the mode of the three $60 \mathrm{~s}$ periods of acoustic injection with a $60 \mathrm{~s}$ pause in between.

The recording of the galactic background radio emission in the absence of infrasound excitation (see Fig. 5a) was characterized by a fairly uniform level of background radio emission across the band of $20-30 \mathrm{MHz}$.

After $\sim 4.5 \mathrm{~min}$ from the start of the infrasound excitation (yellow arrows in Fig. 5b), corresponding to the time of the vertical propagation of the acoustic wave to the heights of the lower ionosphere, $z>80 \mathrm{~km}$, an increase in intensity of galactic radio background was observed. We assume that the cause of this phenomenon is the increase in the ionospheric plasma transparency for the HF radio emission of galactic radio sources during its propagation through the ionosphere (Koshovyy, 1999; Kotsarenko et al., 1999).

After $\sim 40 \mathrm{~min}$ from the beginning of the infrasound radiation (07:46:00-07:56:00 UT), the emergence of the second electromagnetic response was observed. Some possible mechanism of this effect is discussed in Sect. 4.4.

The results of the observations of the artificial acoustic modification of the ionosphere obtained in the experiment 28 November 2013 are in good agreement with investigations provided by the Lviv Centre of the Institute of Space Research and the Physical-Mechanical Institute, Ukraine, of artificial acousto-ionospheric disturbances during November and December 1996 and April and October 1997 using the radio telescope URAN-3 at $25 \mathrm{MHz}$ and the controlled ground-based acoustic generator (Kotsarenko et al., 1999; Koshovyy and Soroka, 1998; Koshovyy et al., 2005).

Measurements of the galactic radio emission have been obtained by the correlation radiometer of the HF radio telescope URAN-3 at $25 \mathrm{MHz}$ as illustrated in Fig. 6. The pulsed bursts of radio signals were revealed by processing the data registered during 24 sessions of the measurements. The bursts have been identified as a reaction of the ionosphere to the infrasound excitation (see Fig. 6). The time delay of the ionospheric response relative to the start of the acoustic sounding is the main evidence in this method. 


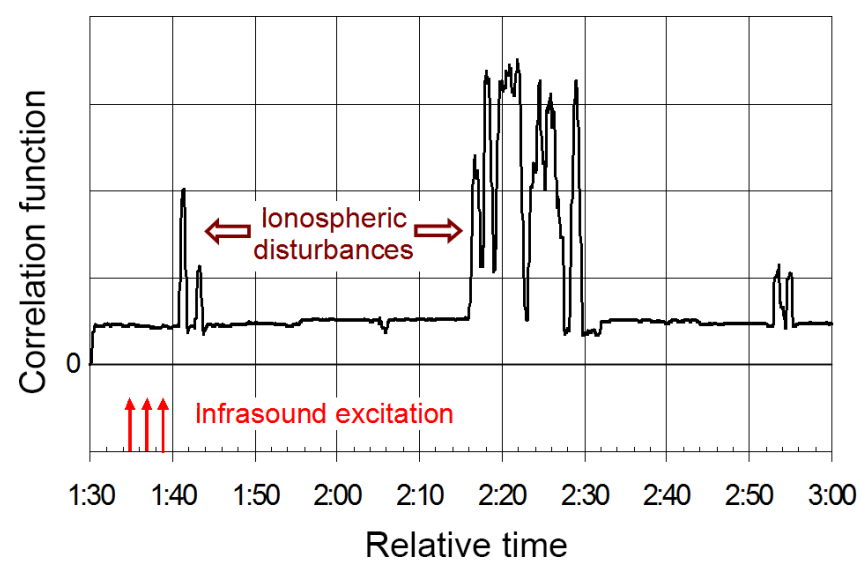

Figure 6. Experimental record of the galactic radio emission has been observed with the correlation radiometer of the HF radio telescope URAN-3 at $25 \mathrm{MHz}$ (1997). The figure shows a module of the mutual correlation function $\left|r_{12 B}(t)\right|$ between the experimental records of the passed radio emission of a space source registered by the first and second half of the RT URAN-3 antenna in miniinterferometer mode with polarization B (linear horizontal). The group of red arrows represents the time model of acoustic excitation. After $\sim 5.5 \mathrm{~min}$ from the start of the infrasound excitation an increase in intensity of galactic radio background is observed.

According to the statistical processing of these results, a series of the time delay variations in the detected atmospheric effects have been obtained: $5.8 \pm 0.5 \mathrm{~min}, 20.7 \pm 2.3 \mathrm{~min}$, $29.3 \pm 1.3 \mathrm{~min}, 41.7 \pm 2.3 \mathrm{~min}$, and $59.6 \pm 4.2 \mathrm{~min}$. These results are shown in Fig. 7 as the histogram describing the time delay variations in the detected ionospheric effects. In the experiment of 28 November 2013 the time delays corresponding to the first $(T \approx 4.5 \mathrm{~min})$ and fourth $(T \approx 40 \mathrm{~min})$ estimates obtained earlier have been registered.

The results of the statistical processing in MATLAB are presented in Table 1 . Here $\bar{X}$ is the unbiased and consistent estimator of the sample mean, $a$ is the probability distribution centre, $S^{2}$ is the unbiased and consistent estimator of the sample variance, $\sigma$ is the mean square deviation, and $P$ is the reliability.

The time delays of the revealed ionospheric disturbances steadily correlate with the propagation time of the sound packet up to the ionosphere. We have interpreted this as evidence that the ground-based acoustic generator creates the artificial acousto-ionospheric disturbances. To confirm this, the following parameters were taken into account:

a. the differences in recordings of galactic radio source signals in the presence of the acoustic excitation compared with the same measurements without it;

b. the first reaction of the ionosphere taking place with a time delay that corresponds to the time of vertical propagation of this wave to the altitudes of the order of $>80 \mathrm{~km}$;

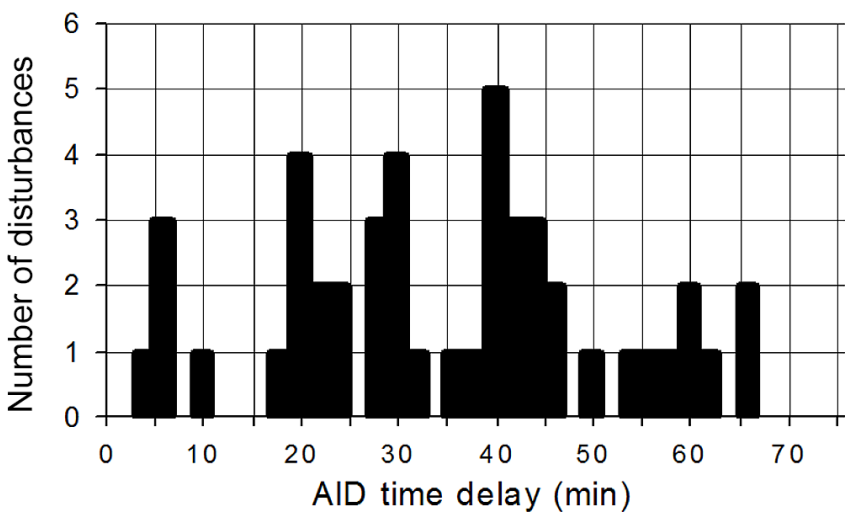

Figure 7. A histogram of the time delay of the ionospheric response relative to the start of the acoustic generation. According statistical processing results, a series of time delay variations in the detected atmospheric effects have been obtained: $5.8 \pm 0.5 \mathrm{~min}$, $20.7 \pm 2.3 \mathrm{~min}, 29.3 \pm 1.3 \mathrm{~min}, 41.7 \pm 2.3 \mathrm{~min}$, and $59.6 \pm 4.2 \mathrm{~min}$ (AID - acoustically generated ionospheric disturbances).

c. the repetition of the ionosphere reactions across a relatively stable series of time delays: 5, 20, 30, 40, and $60 \mathrm{~min}$;

d. the reproducibility of the time pattern of the ionospheric reaction for various galactic radio sources.

The delay time of the ionosphere response to acoustic signals from the ground generator occurs in one or more of the ranges, as we measured. However, the time lag between switching on the generator and the appearance of a response from the signal from a third party would be random. Moreover, in view of the independence (lack of physical links) of such events, we can assume an equal probability for the occurrence of small delays (e.g. equal to zero) as well as large delays (e.g. equal to $24 \mathrm{~h}$ ). A histogram of the time delay variations was constructed relative to the time sequence of switching on the radiator. Although this sequence is selected randomly (on different days, at different times of the day), the delays nonetheless turned out to be grouped in each case. Grouping is present in these cases and reveals the presence of a non-random component in the delays. If the radiator is absent (or is off), we must select an event with respect to which we will determine the delay. We note that, in almost all experiments carried out, a calibration session was carried out before in which the same measurement was made with the generator switched off. Therefore, a direct comparison of the measurement results of the transmitted electromagnetic field in cases of the presence and absence of influence of the parametric acoustic generator on the ionosphere can be made using Fig. 5a and $b$. 
Table 1. Time delay variations in the detected atmospheric effects: confidence intervals for the probability distribution $a$ and mean square deviations $\sigma$, in minutes.

\begin{tabular}{lrrrrr}
\hline No. & $\bar{X}$ & $S$ & $P$ & Confidence intervals for $a$ & Confidence intervals for $\sigma$ \\
\hline 1 & 5.75 & 0.5 & $95 \%$ & $4.83<a<6.67$ & $0.283<\sigma<1.865$ \\
2 & 20.7 & 2.24 & $95 \%$ & $18.84<a<22.49$ & $1.51<\sigma<4.29$ \\
3 & 29.3 & 1.25 & $95 \%$ & $28.03<a<30.54$ & $0.807<\sigma<2.76$ \\
4 & 41.7 & 2.27 & $95 \%$ & $40.36<a<43.07$ & $1.64<\sigma<3.65$ \\
5 & 59.6 & 4.16 & $95 \%$ & $56.17<a<62.94$ & $2.81<\sigma<7.98$ \\
\hline
\end{tabular}
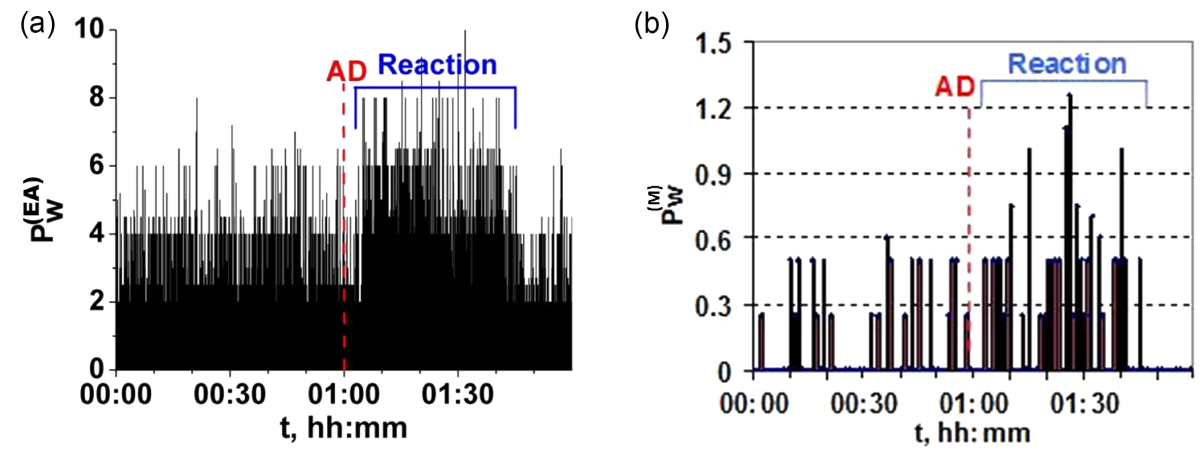

Figure 8. Time distribution of the whistler density before (00:00-01:00) and after (01:00-02:00) the acoustic disturbance (time is measured relative to the beginning of epoch). AD is the moment of acoustic disturbance. Distribution $\left(P_{W}^{(\mathrm{EA})}\right)($ Fig. $8 \mathrm{a})$ was created with the superposed epoch analysis method for data from 32 experiments, carried out on different days. Distribution $\left(P_{\mathrm{W}}^{(\mathrm{M})}\right)(\mathbf{b})$ was created with the median filtration method for the same data.

\subsection{Influence of acoustic atmosphere disturbances on the VLF wave propagation}

It is known that the lower boundary of the ionosphere acts as a shield for the VLF range of $3-30 \mathrm{kHz}$ electromagnetic wave propagation. Whistlers are natural representatives of VLF electromagnetic waves. They are created when electromagnetic pulses emitted by lightnings enter the magnetic flux tube of the Earth's magnetosphere (ducting). Electromagnetic pulses passing through magnetic flux tubes are subject to the dispersive transformation or dissolution into the frequency set, where lower frequencies propagate slower than higher frequency waves (Helliwell, 1965). In the Lviv Centre of the Institute of Space Research, a study of acoustic disturbances in the atmosphere interacting with VLF electromagnetic waves passing through the ionosphere from above was conducted. Signals were recorded by a ground-based VLF receiver and analysed by comparing the whistler density occurring throughout $1 \mathrm{~h}$ before the acoustic generation was switched on and $1 \mathrm{~h}$ after. Figure 8a, which shows the time distribution of the whistler density before and after the acoustic sounding was switched on, was created with the superposed epoch analysis method (Lühr et al., 1998) for $1 \mathrm{~s}$ accounts for data from 32 experiments, which were carried out in 2004 from 15 February to 20 May during the second half of day. The result of processing has made it possible to distinguish the non-random stable character of the reaction of the ionosphere to the artificial acoustic influence. The time interval from the 5th to the 50th minute after the start of the acoustic sounding is characterized by the whistler density increasing. This suggests an increase in the ionospheric transparency for VLF electromagnetic waves. The $5 \mathrm{~min}$ delay of the response (Fig. 8a) is due to the time span required for the acoustic wave propagation from the Earth's surface to ionospheric altitudes. This further suggests that the acoustic disturbance influences the ionospheric transparency and provides an opportunity for VLF waves to propagate from the magnetosphere to the Earth's surface. To exclude an influence of possible single abnormally large events on the results of the measurements, median filtration (Rabiner, 1978; Arce, 2005) has been applied to the 1 min counts of the intensity of whistlers; see Fig. 8b. The median values of the intensity of whistlers are depicted at the vertical axis from the set of 32 experiments. In Fig. 8a and b, an increase is seen in the intensity of whistlers and the density of their occurrence after the acoustic sounding during approximately $40 \mathrm{~min}$, which can be explained by an increase in the transparency of the ionosphere after the artificial acoustic perturbation.

An increase, after the acoustic impact on the atmosphere, in the number of the whistlers recorded per unit time (for $1 \mathrm{~min}$ ) is evaluated statistically. A statistical significance test of differences between mean values is applied (see in Waer- 
(a)

(b)

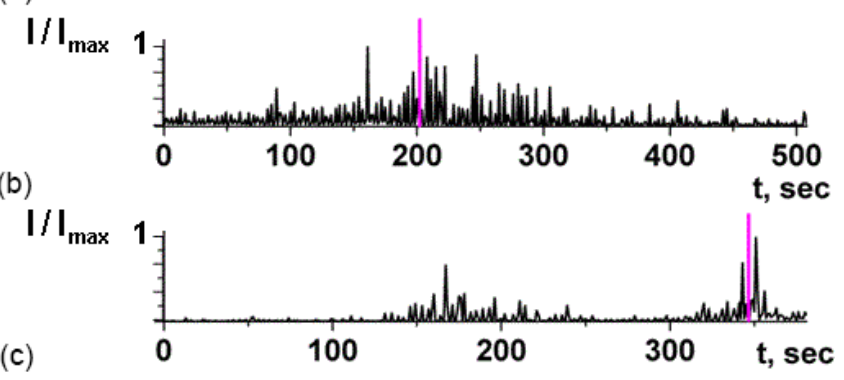

$1 / I_{\max }$

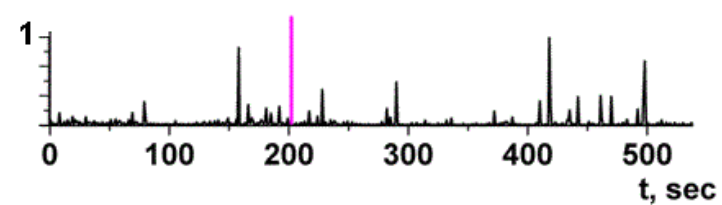

Figure 9. The time distributions of the intensities of VLF range signals registered on board the DEMETER satellite, normalized to the corresponding maximum values: (a) and (b) after the acoustic disturbance, and (c) without the acoustic disturbance. The beginning of the acoustic radiation was chosen at a moment of time $10 \mathrm{~min}$ before the moment of time corresponding to the minimum distance from the satellite to the point of the acoustic generation. The vertical arrow of each plot is the moment of the minimum distance from the satellite to the point of the acoustic generation (Lviv). Panel (a) shows satellite flight over artificial acoustically disturbed zone (200 s), (b) shows satellite flight over Vrancea earthquake zone (Romania) first (130-210 s) and then over artificial acoustically disturbed zone (320-380 s), and (c) shows satellite flight over the point of the acoustic radiator location when generation is absent (200s).

den, 1971). It is concluded that the increase in the number of whistlers after the acoustic impact is of a non-random nature with an estimated probability $\sim 0.99$.

The intensity of VLF waves travelling in the opposite direction (penetrating into the ionosphere from the ground level) should also increase. To investigate this hypothesis, a test observational series with satellite DEMETER has been carried out (Parrot at al., 2007; Soroka at al., 2006). The VLF wave signal from navigational stations RSDN-20 ("Alpha") with a frequency of $12648.809 \mathrm{~Hz}$ has been used as a benchmark. In Fig. 9 the examples of the intensity distribution of VLF signals registered on board DEMETER are shown. The start of the acoustic sounding was chosen to be $10 \mathrm{~min}$ before the bypass of the satellite over PSG. This time is enough for AGWs to reaching the ionosphere. The time moment of the bypass of the satellite is marked by the vertical arrow in Fig. 9a-c. The intensity of VLF waves after the start of the acoustic generation (Fig. 9a, b) is significantly larger than in the absence of the acoustic generation (Fig. 9c). We compare the signals received during the DEMETER satellite flight over the Vrancea earthquake zone in Romania (130$210 \mathrm{~s}$ in Fig. 9b) with satellite signals registered later over the artificial acoustically disturbed zone (320-380 s in Fig. 9b). The comparison shows that natural and artificial acoustical

disturbances are similar in shape, which allows us to assume the similarity of the influence of both disturbance sources on the ionosphere. This result can provide evidence that supports an idea and a new instrument for the study of seismic phenomena using satellite data.

As a result of Sect. 3.2, we conclude that acoustic disturbances in the atmosphere may cause a change in the ionosphere transparency.

\section{Discussion and conclusions}

\subsection{The impact of the parametric acoustic generator on the ionosphere}

We discuss the influence of the acoustic waves excited by a ground-based generator on the ionosphere. Acoustic waves cause an increase in transparency of the electromagnetic waves in HF (MHz) and VLF (kHz) frequency bands. This conclusion is revealed on the basis of experiments with the effect detected from ground-based and satellite instruments.

The sound waves' propagation time to the altitudes of $\mathrm{E}$ region and of the lower edge of the $\mathrm{F}$ region is equal to 5-7 $\mathrm{min}$. It was shown experimentally that the corresponding group delay for electromagnetic waves in the HF range has the smallest delay dispersion. That corresponds to the expected direct influence of the sound waves on the ionosphere without any hypothetical accumulation effects. These direct effects could be, for example, a deformation or altitude shift of corresponding ionospheric layers, which can cause a change in characteristics of the wave propagation.

In addition, the Doppler shift corresponding to the acoustic oscillations was recorded in the ionosphere simultaneously with variations in the electromagnetic HF waves reflected from the ionospheric inhomogeneities. An increase in reflection can be caused by the deformation of existing inhomogeneities and by the shift due to acoustic gravity waves penetrating to the ionospheric altitudes, after transformation of the sound waves generated by the PSG.

The atmospheric acoustic events are accompanied by many changes in external conditions which occur in the real atmosphere during the packet $(60 \mathrm{~s})$ propagation. Then, the total energy released during our experiment $\left(E \sim 10^{4} \mathrm{~J}\right)$ is relatively low in comparison with other known ground sources of acoustic energy, which causes an influence on the ionosphere (Chernogor and Rozumenko, 2008). Therefore, we should not expect that the ionospheric response will be absolutely repeatable in all cases.

The successful experiment outcomes in our study, connected with the transparency increase for electromagnetic waves, depend on a number of factors:

1. the acoustic power of the generator;

2. the difference frequency, which is formed in the area of the parametric antenna; 
3. a time diagram of the signal during its generation;

4. the selected measurement method;

5. the presence and degree of dynamic processes development in the neutral atmosphere and ionosphere.

There were three series of experiments with approximately the same conditions in each series, including 32 experiments with VLF $(\mathrm{kHz})$ electromagnetic waves/whistlers of natural origin (Fig. 8), 20 experiments with VLF (kHz) observations by the DEMETER satellite, and 24 experiments with galactic radio sources (in $\mathrm{MHz}$ range). The total number of active sessions of measurements is 76 cases, when the appearance of disturbances in the ionosphere and its delay determination has been registered. Each active session was preceded by a session of calibration measurements in which the local state of the ionosphere was characterized by the sound generator being turned off.

Some outcomes supporting the general conclusions on influence by AGWs on the ionosphere from the PSG are also drawn from the case studies of (1) registration on the satellite DEMETER of $\mathrm{kHz}$ radiation generated by the ground-based navigation station (Fig. 9) and (2) the changes in reflection of HF electromagnetic waves, injected by a ground transmitter. This effect was accompanied by the recording of the Doppler shift of the reflected electromagnetic wave, caused by the sound wave from the PSG in the ionosphere (Fig. 4).

Note that in the recent ground-satellite measurements by the CHIBIS satellite (Cheremnykh et al., 2014) we were able to analyse the main factors of influence on the local ionosphere. It has been shown that parameters of the transmitted, reflected, and scattered radio signal are well correlated, in particular, in terms of the delay time, with parameters of the acoustic injection, as well as that the experiments recorded weak ionospheric disturbances caused by this radiation.

\subsection{Another possible cause of the registered effects in the ionosphere}

We can consider the possibility that, instead of artificial excitation, we registered the response from natural sources, in particular from lightning. The arguments which support the artificial reason of the ionosphere impact from the PSG are as follows. The PSG creates an acoustic signal of the artificial waveform. It differs from most natural signals in their time characteristics. First, natural sources like thunderstorms have an exposure time less than $1 \mathrm{~s}$, but one packet in PSG signal has a duration of $60 \mathrm{~s}$. Secondly, the PSG creates a series of signals with three packets having the $60 \mathrm{~s}$ interval between submissions. However, the appearance of three lightning strikes with the exact intervals between them of $60 \mathrm{~s}$ is very improbable.

As seen from Fig. 6, time delays in the ionosphere response less than 4 min are practically absent. Based on our even relatively small set of observations we can conclude that the ionospheric disturbance appeared exactly with 5-6 min delay after the PSG start operation. Therefore, due to the special artificial signal waveform, which makes it different from natural and other technical signals, and spatio-temporal characteristics of the ionospheric response, it is possible to assume the existence of an acousto-ionospheric effect created by the PSG.

The ionospheric response to a change in electromagnetic transmittance due to the influence of acoustic waves from the parametric generator is specific and cannot be explained by an influence of the lightning strike. In the VLF waveband (Fig. 8), the time distribution of the number of registered whistlers of a natural origin demonstrates a clear change after activating the acoustic parametric generator with a delay of $6 \mathrm{~min}$. This time corresponds to the delay required for the acoustic gravity waves to reach the $\mathrm{E}$ region.

The possibility that the registered effect of the electromagnetic wave transmission changes could be caused by a powerful natural source, such as an earthquake, is considered as well. This case is possible, in principle, if the launch time of the sound generator coincides with an earthquake both in time and space. This is seen from the comparison between the signals shown in Fig. 9c, which concerns electromagnetic VLF signals from the navigation radio station registered on board the DEMETER satellite. Peaks in Fig. 9, presumably caused by infrasound waves from a seismoactive region and the activation of a parametric generator, are shown in Fig. 9b at relative times 170 and $330 \mathrm{~s}$. Those peaks are of the same order of amplitude and similar in shape (bell-like).

Analysis of the experimental results has been carried out as well with regard to the state of solar activity in order to avoid the effect of solar flares on the ionosphere. Note that the time distribution of ionospheric disturbances caused by natural factors is random, while the responses caused by the acoustic injector have stable time delays relative to the time of the acoustic wave generation.

\subsection{Correspondence between the theoretical estimations and the wave effects in the ionosphere}

The simulations and experiments have demonstrated a high efficiency in the generation of AGWs due to a cascade scheme of the double frequency transform $600 \mathrm{~Hz} \rightarrow 25 \mathrm{~Hz} \rightarrow 0.016 \mathrm{~Hz}$. The simulations have been conducted for the parameters of the acoustic generator. Wave diffraction is the factor that limits the efficiency of this transform. To avoid those limitations we propose to use the higher intermediate frequency $\sim 100 \mathrm{~Hz}$ or to increase the initial widths of the $\mathrm{SW}$ beam to $\sim 10 \mathrm{~m}$. The amplitudes of AGWs in the ionosphere increase as the square of the initial amplitudes of the sound waves $v_{0}$ at $600 \mathrm{~Hz}$, when these amplitudes are $v_{0} \leq 300 \mathrm{~m} \mathrm{~s}^{-1}$. At higher initial amplitudes, the saturation of the increase in the amplitudes of AGWs occurs, due to the intense generation of harmonics of the sound waves at small heights $z<0.05 \mathrm{~km}$. 
The present model is quite different from the previous models of the penetration of sound waves from the lower atmosphere to ionospheric altitudes. In particular, in contrast to Krasnov and Kuleshov (2014), (1) effects of gravity are taken into account and (2) two-stage frequency transforms in two ranges of altitudes (600-20 and $20-0.01 \mathrm{~Hz}$ at $0-100 \mathrm{~m}$ and from $100 \mathrm{~m}$ to $\sim 20 \mathrm{~km}$ ) and the propagation of AGWs from $\sim 20 \mathrm{~km}$ to ionospheric altitudes have been considered. It is shown that acoustic waves are strongly nonlinear sound waves at the input of the system (at $Z=0 \mathrm{~km}$ ) and generate practically linear atmospheric acoustic gravity waves at the ionospheric altitudes. The results of modelling correspond well to the Doppler shift recorded simultaneously with the change in intensity of HF electromagnetic waves reflecting from some inhomogeneities in the ionosphere. The experimental and theoretical results correspond to each other in the following ways: an increase in the measured intensity of the electromagnetic waves reflected from the ionosphere (Fig. 4a), the delay time from the sound waves launched from the ground generator (Fig. 1), the duration of the modulation pulse $(1 \mathrm{~min})$, the value and the duration of the measured Doppler shift (Fig. 4b), and the results based on the developed model for the velocity of the medium and corresponding Doppler shift (Fig. 4c) correspond to each other. Our hypothesis is that the observed change in the reflection from the ionospheric inhomogeneities is caused by the deformation of corresponding inhomogeneity caused by AGWs. The acoustic double frequency transform has been simulated. The initial SW frequencies are $f_{1}=110 \mathrm{~Hz}$ and $f_{2}=100 \mathrm{~Hz}$ as in the experiments detailed above. The initial amplitude of the SW is $200 \mathrm{~m} \mathrm{~s}^{-1}$, the width of the beam is $2 \mathrm{~m}$, as in that experiment. The intermediate frequency is $f=f_{1}-f_{2}=$ $10 \mathrm{~Hz}$. The ULF frequency is $F=0.016 \mathrm{~Hz}$. The Doppler frequency shift has been estimated from the formula $F_{\mathrm{D}}=$ $2 f_{\text {radio }}\left(V_{\text {max }} / c\right) \sin \theta_{i}$, where $f_{\text {radio }}=16.173 \mathrm{MHz}, V_{\max }$ is the maximum velocity of the air for the ULF sound wave, and $\theta_{I}=38.6^{\circ}$ is the incidence angle of the radio signal to the ionospheric inhomogeneity. Essential Doppler frequency shifts occur at the altitudes $Z \geq 150 \mathrm{~km}$, as seen from Fig. $4 \mathrm{a}$. At the altitude $Z=160 \mathrm{~km}$ the Doppler frequency shift is about $F_{\mathrm{D}} \sim 3 \mathrm{~Hz}$. The experimental data are $F_{\mathrm{D}}=3-7 \mathrm{~Hz}$, as seen from Fig. 4 b. Therefore, the simulation corresponds to the experimental results.

Experimental investigations of artificial acoustoionospheric disturbances were also conducted using the broadband registration system of the radio telescope URAN3 , which records the dynamic spectra of $\mathrm{HF}$ range, or decametre wave range of the radio emission from galactic radio sources.

In the experiments described in Sect. 3 of the present paper, the observed minimum time delay of 4 to $7 \mathrm{~min}$ corresponds to the time taken for signal propagation to $80-130 \mathrm{~km}$ altitudes (see Fig. 1). Our assumption is that an influence of AGWs on the ionosphere $\mathrm{E}$ region with the minimum time delay may be caused by some relatively low-inertial direct mechanism such as shifting or deformation of some ionospheric inhomogeneity, without more inertial hypothetical accumulation effects. This assumption is supported by the fact that the corresponding delay in the response to HF waves in the ionosphere is characterized by the minimum dispersion (see Fig. 7 and Table 1). The modulation of the intensity of the radio waves $(20-30 \mathrm{MHz})$ and the variations in the intensity of whistlers $(3-30 \mathrm{kHz})$, both coming from above, can be explained by a change in the electron concentration within the E layer produced by the acoustic signal. The width of the infrasound beams in the E layer will rise to $\sim 5 \mathrm{~km}$, which is comparable with the wavelengths of whistlers: $2 \pi\left(c / \omega_{\mathrm{pe}}\right)\left(\omega_{\mathrm{He}} / \omega\right)^{1 / 2}$, where $\omega_{\text {pe }}$ and $\omega_{\mathrm{He}}$ are the electron plasma and cyclotron frequencies. For the E layer, the whistler wavelengths are in the range $0.5-10 \mathrm{~km}$. Therefore, at least a dimension of the expected region with changed electron concentration due to acoustic waves is suitable for the creation of a lens or a deflector for whistlers, as well as for the galactic radio waves. Another possibility for the temporary increase in the ionosphere transparency for the galactic radio waves is the creation of a spatial dynamic grating due to AGW modulation of the neutral gas, as considered in Kotsarenko et al. (1999). A ULF AGW modulates the density of the ionosphere (Koshevaya et al., 2005) and the modulation values can be estimated as $n^{\prime} / n_{0} \sim v_{z} / c_{\mathrm{s}}$, where $v_{z}$ is the air velocity in an $\mathrm{AGW}$ and $c_{\mathrm{s}}$ is the sound velocity. In turn, due to the modulation of the plasma density within $\mathrm{F}$ layer, it is possible to obtain the changes in the transparency of the radio waves of about $5 \%$ for the air velocities at $z=300 \mathrm{~km}$ presented in Fig. 2. The frequencies of the modulated radio waves are chosen near the lower edge of the transparency band 15-20 MHz. Therefore, we obtain the qualitative agreement between theoretical and experimental results.

Concerning the ionospheric responses with a time delay of about 10-30 min, these may presumably be connected with the processes in the $\mathrm{F}$ region of the ionosphere such as the photochemistry processes and the release of energy accumulated in the atmosphere-ionosphere system or dissipation (Emelyanov et al., 2015; Pulinets et al., 2011; Sorokin and Hayakawa, 2014). Another effect concerning a possible delay in the ionospheric response could be re-reflections from the boundaries of the "magnetosphere-ionosphere" or "ionosphere-atmosphere" during bounce oscillations.

For the considered model of the acoustic generator the amplitudes of the air displacements within the resulting AGW are $v / \Omega \sim 10 \mathrm{~m}$ in the F layer at $300 \mathrm{~km}$ (see Fig. 2). These displacements are too small to cause the direct modulation of galactic radio waves. Nevertheless, using the wider initial SW beams at a frequency of $600 \mathrm{~Hz}$ it is possible to obtain higher values of displacements, which could lead to observable effects, like the increase in F-layer transparency. 


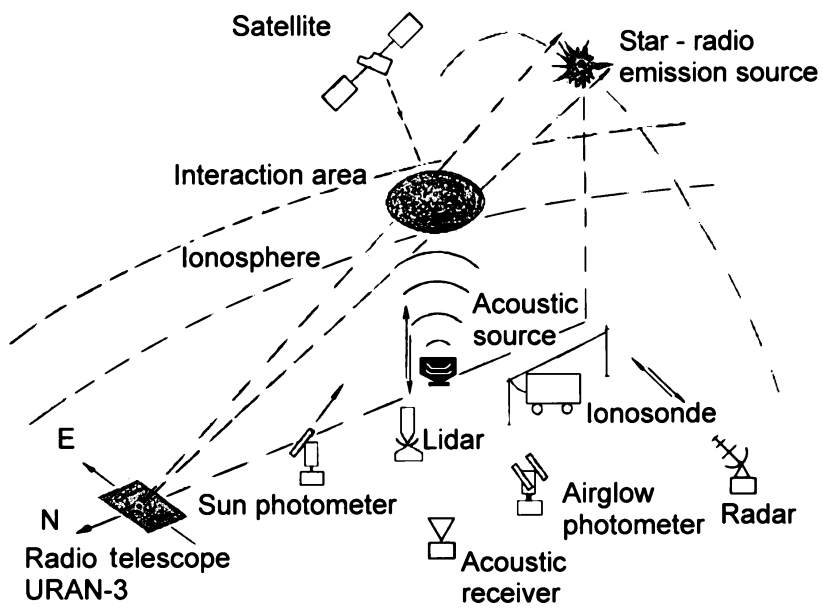

Figure 10. Acoustic active experiment proposed for verification of the model and mechanisms of the influence of the acoustic soundings on the ionosphere. Acoustic generator (Acoustic source) will operate at two close frequencies to produce impact at the Interaction area in the ionosphere. The comprehensive set of ground-based and satellite detectors, which includes an ionosonde, atmospheric lidar, radio telescope URAN-3, airglow photometer, and aerosol sun photometer, will register background conditions and expected ionospheric disturbances produced by the acoustic wave.

\subsection{Active acoustic experiment for acoustic soundings' influence on the ionosphere}

On the basis of this and previous works, we would like to propose an idea for a multiple comprehensive acoustic active experiment. We have demonstrated (see Sect. 3) that an influence on the ionosphere of the artificial sound wave radiated by the ground-based PSG is detectable, in spite of the fact that the energy release $(W)$ in our experiments $(W \sim$ $10^{4} \mathrm{~J}$ ) is comparatively less than in the powerful MASSA experiment, rocket launch, or nuclear explosions (with $W$ of the order of $10^{9}, 10^{13}$, and $10^{17} \mathrm{~J}$, respectively), where acoustic-electromagnetic energy transformations take place (Gokhberg and Shalimov, 2000; Chernogor and Rozumenko, 2008).

The experiment would include the impact from a groundbased acoustic generator towards the ionosphere, which includes the measurements of associated electromagnetic and acoustic fields, effects in aerosols on the sound wave attenuation/amplification, optical measurements of associated atmospheric glows, and radio physical measurements of the attenuation/amplification of emissions from galactic radio sources with a set of ground-based instrumentation. During the experiment (see Fig. 10) the acoustic generator will operate at two close frequencies. The signal at the differential frequency is within the infrasound acoustic wave band and, due to the nonlinear interaction in the atmosphere, propagates to the ionosphere. The acoustic receiver (acoustic spectral analyser) located at the same distance from the source detects the scatter by the atmospheric acoustic waves. The interaction area is the ionospheric local region disturbed by acoustic waves. The HF ionospheric vertical sounder (ionosonde) will be used to obtain ionospheric electron concentration measurements up to the F layer. The atmospheric light radar (lidar) will be used to measuring tropospheric parameters including aerosol height distribution and acoustic wave disturbances in the atmosphere. The radio telescope URAN-3 will provide measurements of radio emissions from sky radio sources (astronomical sources of radio emission including quasars and pulsars) in the HF band and ionospheric disturbances, including an influence of acoustic waves on radio wave propagation. The radio transmitter/receiver (radar in Fig. 10) allows detection of possible disturbances in the upper atmosphere/ionosphere. The aerosol sun photometer provides registration of the total aerosol content in the atmosphere when using the Sun's radiation, and the optical scanning photometer (airglow photometer) and low-light-level TV camera will record optical emissions in the lower ionosphere. Satellite instruments will detect (in situ and remotely) the atmospheric and ionospheric disturbances produced by acoustic waves.

The goal of the parametric two-frequency acoustic generator experiment is to check in practice the theoretical assumption on propagation and dissipation of acoustic waves in the atmosphere. The first step in the experiment development is the detailed study of the properties of the acoustic source. We have to estimate the frequency spectrum of the generator and its acoustic power to map velocity and pressure fields and to provide a detailed study of nonlinear transformation of frequencies in the atmosphere. When the frequency combination is generated, it is important to determine the power of radiation at the differential ISW frequency. The next step is the detailed study of the possible influence of the acoustic emission on the atmospheric and ionospheric constitutions. In previous experiments the change in the atmospheric aerosols state was measured under the influence of the acoustic source radiation. The aerosol sun photometer is suitable to study the influence of acoustic waves on atmospheric aerosols, if we expect aerosol optical thickness variations (Milinevsky et al., 2012; Bovchaliuk et al., 2013; Danylevsky et al., 2011). The ionosonde will be helpful for the study of ionospheric modification, as well as the diagnostics of the ionosphere by the URAN-3 HF radio telescope using astronomical radio sources. The study of the ionospheric emissions in visual and near-infrared spectral bands by photometers and lowlight-level TV cameras will allow us to detect optical phenomena in the acoustic wave injection. The low-frequency radar and GPS satellite signals will provide additional information on ionospheric disturbances and the ionospheric electron concentration. In situ measurements from satellites at ionospheric altitudes will be important for diagnostics of the near-Earth environment. In general, the proposed future experiment would be able to verify a theoretical model and 
mechanisms of the influence of the acoustic soundings on the ionosphere.

\subsection{Final conclusions}

The influence on ionospheric transparency for the electromagnetic VLF and HF wave caused by the parametric soundgenerator-excited acoustic waves has been observed in a number of experiments. This effect was observed for electromagnetic waves artificially excited from the ground and detected at ground-based observatories and satellites in different seasons, days, and times of day. Due to a special artificial signal waveform, which helps to separate it from natural signals, we can consider measured spatio-temporal characteristics of the ionospheric response as the influence of artificial injection. Our analysis shows that the other possible reasons for the observed increase in the ionospheric transparency for VLF and HF waves are practically excluded.

The new model of the penetration of acoustic waves excited by a ground-based PSG up to the ionospheric altitudes has been developed. It is shown that the finite size of the initial aperture, diffraction, dissipation, dispersion, and nonlinearity of the beam determines the characteristics of the AGW, which is finally able to reach the altitudes of the $\mathrm{E}$ and $\mathrm{F}$ regions.

The time delay of the ionospheric response to the excitation of sound waves by the PSG, as well as the value of the Doppler shift of the HF electromagnetic waves reflected from the ionosphere, has been determined on the basis of the developed nonlinear model. The above-mentioned characteristics correspond well to those obtained experimentally. The modulation of the intensity of the radio waves at frequencies between 20 and $30 \mathrm{MHz}$ and the variations in the intensity of whistlers $(3-30 \mathrm{kHz})$, both coming from above, can be explained by a change in the electron concentration within the E layer produced by the acoustic signal. At least a dimension of the region with changed electron concentration is suitable for the creation of a lens or a deflector for whistlers, as well as for the galactic radio waves. The AGW influence on the ionosphere has been estimated for the cases of the modulation of the ionospheric plasma concentration. The latter effect is shown as an alternative possible reason of the increasing of the ionosphere transparency for the propagation of HF waves.

Some mechanisms of direct and relatively fast impact of the AGW on the ionosphere (such as shift or deformation of the ionospheric inhomogeneities) and corresponding indirect and more inertial impact (such as accumulation) have been mentioned as hypothetical. Due to the complexity of the proposed nonlinear model of the PSG-injected acoustic wave transformation into AGWs and their penetration to ionospheric altitudes, we limited our consideration by simulation of the neutral perturbations only.

The active experiment for investigation of the artificial sound waves impact on the atmosphere-ionosphere system is proposed (in Sect. 4.4). Comprehensive observations during the active experiment with acoustic soundings and our theoretical model would be useful for improving the effectiveness of the controllable acoustic influence on the ionosphere and understanding the mechanisms of seismo-ionospheric coupling. The proposed future experiment based on the developed theoretical model would be able to verify the model results and the mechanisms of the influence of the acoustic waves on the ionosphere. In order to explain some ionospheric phenomena due to a controlled acoustic impact, the proposed theory needs further development and experimental verification, and this is the subject of further research.

In conclusion, based on previous experiments and computer simulation, we can see evidence that the increase in ionosphere transparency for the HF and VLF electromagnetic waves could be caused by the PSG-injected acoustic wave impact on the ionosphere as a result of cascade transformations.

\section{Data availability}

Original experimental data are available by request to the following participants of the experimental teams conducting corresponding experimental studies, via e-mail: in particular, for data on HF measurements contact Oleh L. Ivantishin (o.ivantyshyn@gmail.com), and for data on VLF measurements contact Roman T. Nogach (r.t.nogach@ gmail.com) or Valentyn P. Mezentsev (m5z5ncev@gmail.com).

Acknowledgements. This publication is based on work supported in part by STCU Project 6060 . The work was also partly supported by project 16BF051-02 of the Taras Shevchenko National University of Kyiv and by a grant of the State Fund for Fundamental Research, project F73/115-2016. Viktor N. Fedun would like to acknowledge the Royal Society for support received.

The topical editor, Christoph Jacobi, thanks O. A. Pokhotelov and the three anonymous referees for their help in evaluating this paper.

\section{References}

Aramyan, A. R., Galechyan, G. A., Harutyanyan, G. G., Mangasaryan, N. R., Bilen, S. G., and Soroka, S.: Modeling of interaction of acoustic waves with ionosphere, IEEE Trans. Plasma Sci., 36, 305-309, 2008.

Arce, G. R.: Nonlinear signal processing: A statistical approach, Wiley: New Jersey, USA, 607 pp., 2005.

Bovchaliuk, A., Milinevsky, G., Danylevsky, V., Goloub, P., Dubovik, O., Holdak, A., Ducos, F., and Sosonkin, M.: Variability of aerosol properties over Eastern Europe observed from ground and satellites in the period from 2003 to 2011, Atmos. Chem. Phys., 13, 6587-6602, doi:10.5194/acp-13-6587-2013, 2013. 
Cheremnykh, O. K., Klimov, S. I., Korepanov, V. E., Koshovy, V. V., Melnik, M. O., Ivantyshyn, O. L., Mezentsev, V. P., Nogach, R. T., Rapoport, Yu. G., Selivanov, Yu. A., and Semenov, L. P.: Ground-space experiment for artificial acoustic modification of ionosphere. Some preliminary results, Space Sci. Technol., 20, 60-74, doi:10.15407/knit2014.06.060, 2014.

Chernogor, L. F. and Rozumenko, V. T.: Earth-atmospheregeospace as an open nonlinear dynamical system, Radio Phys. Radio Astron., 13, 120-137, 2008.

Danylevsky, V., Ivchenko, V., Milinevsky, G., Grytsai, A., Sosonkin, M., Goloub, P., Li, Z., and Dubovik, O.: Aerosol layer properties over Kyiv from AERONET/PHOTONS sun photometer measurements during 2008-2009, Int. J. Remote Sens., 32, 657-669, doi:10.1080/01431161.2010.517798, 2011.

Emelyanov, L. Ya., Zivolup, T. G., Soroka, S. A., Cheremnykh, O. K., and Chernogor, L. F.: Ground acoustic influence on the ionosphere: the results of observations by means of incoherent scattering and vertical sounding the methods, Radio Phys. Radio Astron., 20, 37-47, 2015.

Genzano, N., Aliano, C., Filizzola, C., Pergola, N., and Tramutoli, V.: A robust satellite technique for monitoring seismically active areas: The case of Bhuj-Gujarat earthquake, Tectonophysics, 431, 197-210, 2007.

Gokhberg, M. B. and Shalimov S. L.: Lithosphere-ionosphere coupling and its modeling, Russian J. Earth Sci., 2, 95-108, 2000.

Grimalsky, V. V., Kremenetsky, I. A., and Rapoport, Yu. G.: Excitation of electromagnetic waves in the lithosphere and their penetration into ionosphere and magnetosphere, J. Atmos. Electr., 19, 101-117, 1999.

Grimalsky, V. V., Koshevaya, S. V., Perez-Enriquez, R., and Kotsarenko, A. N.: Nonlinear excitation of ULF atmosphereionosphere waves and magnetic perturbations caused by ELF seismic acoustic bursts, Phys. Scripta, 67, 453-456, 2003.

Hayakawa, M.: Earthquake Prediction with Radio Techniques, Wiley \& Sons, Singapore, 294 pp., 2015.

Helliwell, R. A.: Whistlers and Related Ionospheric Phenomena, Stanford University Press, USA, 349 pp., 1965.

Iyemori, T., Nakanishi, K., Aoyama, T., Yokoyama, Y., Koyama, Y., and Luhr, H.: Confirmation of existence of the small-scale fieldaligned currents in middle and low latitudes and an estimate of time scale of their temporal variation, Geophys. Res. Lett., 42, 22-28, doi:10.1002/2014GL062555, 2015.

Jursa, A. S. (Ed.): Handbook of Geophysics and Space Environment, Air Force Geophys. Lab., US Air Force, 1023 pp., 1985.

Kafatos, M., Ouzounov, D., Pulinets, S., Cervone, G., and Singh, R.: Energies associated with the Sumatra Earthquakes of December 26, 2004 and March 28, 2005, AGU 2007 Fall Meeting, San Francisco, S42B-04, 1-4, 2007.

Klimenko, M. V., Klimenko, V. V., Zakharenkova, I. E., Pulinets, S. A., Zhao, B., and Tsidilina, M. N.: Formation mechanism of great positive TEC disturbances prior to Wenchuan earthquake on May 12, 2008, Adv. Space Res., 48, 488-499, 2011.

Koshevaya, S., Burlak, G., Grimalsky V., Perez-Enriquez, R., and Kotsarenko, A.: Nonlinear Transformation of Seismic waves Into ULF Atmospheric Acoustic Waves, System Analysis Model, Simulation (SAMS), 42, 261-268, 2002.

Koshevaya, S. V., Grimalsky, V. V., Perez-Enriquez, R., and Kotsarenko, A. N.: Increase of the transparency for cosmic radio waves due to the decrease of density of the ionosphere caused by acoustic waves, Phys. Scripta, 72, 91-99, 2005.

Koshovy, V. V., Lozynsky, A. B., Romanchev, Y. V., and Ivantyshyn, O. L.: Radiophysical complexes for cosmic investigations based on Ukrainian radiotelescope URAN-3, Acta Cosmo., 23, 67-70, 1997.

Koshovyy, V., Nazarchuk, Z., Romanyshyn, I., Ivantyshyn, O., Lozynskyy, A., Soroka, S., and Alyokhina, L.: AcoustoElectromagnetic Investigations of an Acoustical Channel of the Lithosphere-Ionosphere Interaction, in: Proceedings of the XXVIIIth General Assembly of International Union of Radio Science (URSI), 23-29 October 2005, New Delhi, India, 4 pp., 2005.

Koshovyy, V. V.: Radiophysical and radio astronomical diagnostics of ionospheric effects induced by a ground-based infrasonic transmitter (preliminary results), Radiophys. Quantum Electron., 42, 691-704, 1999.

Koshovyy, V. V. and Ivantyshyn, O. L.: Detecting of the weak acousto-ionospheric disturbances by the radioscattering method using decameter radio telescope URAN-3, Inf. Extract. Process., 12, 32-36, 1998.

Koshovyy, V. V. and Ivantyshyn, O. L.: Research of the Artificial Acousto-Ionosperic Disturbances, XXVIth General Assembly of the International Union of Radio Science, 13-21 August 1999, Toronto, Canada, Toronto, University of Toronto, 506 pp., 1999.

Koshovyy, V. V. and Soroka, S. O.: Acoustic excitation ionospheric plasma by ground-based radiator. I. Experimental detection of acousto-ionospheric disturbances, Space Sci. Technol., 4, 3-17, 1998.

Kotsarenko, N., Soroka, S., Koshevaya, S., and Koshovy, V.: Increase of the transparency of the ionosphere for cosmic radiowaves caused by a low frequency wave, Phys. Scripta, 59, 174-181, 1999.

Krasnov, V. M. and Kuleshov, Yu. V.: Variation of infrasonic signal spectrum during wave propagation from Earth's surface to ionospheric altitudes, Acoust. Phys., 60, 19-28, 2014.

Liu, J. Y., Chuo, Y. J., Shan, S. J., Tsai, Y. B., Chen, Y. I., Pulinets, S. A., and Yu, S. B.: Pre-earthquake ionospheric anomalies registered by continuous GPS TEC measurements, Ann. Geophys., 22, 1585-1593, doi:10.5194/angeo-22-1585-2004, 2004.

Lühr, H., Rother, M., Iyemori, T., Hansen, T. L., and Lepping, R. P.: Superposed epoch analysis applied to large-amplitude travelling convection vortices, Ann. Geophys., 16, 743-753, doi:10.1007/s00585-998-0743-0, 1998.

Milinevsky, G. P., Danylevsky, V. O., Grytsai, A. V., Evtushevsky, O. M., Kravchenko, V. O., Bovchaliuk, A. P., Bovchaliuk, V. P., Sosonkin, M. G., Goloub, Ph., Savitska, L. Y., Udodov, E. V., and Voytenko, V. P.: Recent development of atmosphere research in Ukraine, Adv. Astron. Space Phys., 2, 114-120, 2012.

Molchanov, O. A., Hayakawa, M., and Rafalsky, V. A.: Penetration characteristics of electromagnetic emissions from an underground seismic source into the atmosphere, ionosphere, and magnetosphere, J. Geophys. Res., 100A, 1691-1712, 1995.

Ouzounov, D., Liu, D., Chunli, K., Cervone. G., Kafatos, M., and Taylor, P.: Outgoing long wave radiation variability from IR satellite data prior to major earthquakes, Tectonophysics, 431, 211-220, 2007.

Parrot, M., Hayosh, M., and Soroka, S. A.: Acoustic experiments in the ionosphere with the DEMETER satellite, EGU General 
Assembly, Vienna, 15-20 April 2007, 1607-7962/gra/EGU2007A-04428, 2007.

Pulinets, S.: The synergy of earthquake precursors, Earthq. Sci., 24, 535-548, 2011.

Pulinets, S. and Boyarchuk, K.: Ionospheric Precursors of Earthquakes, Berlin, Springer, 315 pp., 2004.

Rabiner, L.: Theory and applications of the digital signal processing, Moscow, Mir, 848 pp., 1978.

Rapoport, V. O., Bespalov, P. A., Mityakov, N. A., Parrot, M., and Ryzhov, N. A.: Feasibility study of ionospheric perturbations triggered by monochromatic infrasonic waves emitted with a ground-based experiment, J. Atmos. Sol.-Terr. Phys., 66, 10111017, 2004.

Rapoport, Yu., Selivanov, Yu., Ivchenko, V., Grimalsky, V., Tkachenko, E., Rozhnoi, A., and Fedun, V.: Excitation of planetary electromagnetic waves in the inhomogeneous ionosphere, Ann. Geophys., 32, 449-463, doi:10.5194/angeo-32-449-2014, 2014a.

Rapoport, Yu., Selivanov, Yu., Ivchenko, V., Milinevsky, G., Grimalsky, V., and Tkachenko, E.: The new models of electromagnetic and hydromagnetic wave processes in the ionosphere. Possible applications to coupling phenomena in LAIM/MIAL system, in: Book of Abstract of Annual International Conference "Astronomy and Space Physics", Kyiv, Ukraine, 2730 May 2014, 95-96, 2014b.

Rapoport, Yu. G., Gotynyan, O. E., Ivchenko, V. M., Kozak, L. V., and Parrot: M.: Effect of acoustic-gravity wave of the lithospheric origin on the ionospheric $\mathrm{F}$ region before earthquakes, Phys. Chem. Earth, 29, 607-616, 2004a.

Rapoport, Yu. G., Hayakawa, M., Ivchenko, V., Juarez-R., D., Koshevaya, S., and Gotynyan, O.: Change of ionospheric plasma parameters under the influence of electric field which has lithospheric origin and due to radon emanation, Phys. Chem. Earth, 29, 579-587, 2004b.
Rapoport, Yu. G., Hayakawa, M., Gotynyan, O. E., Ivchenko, V. N., Fedorenko, A. K., and Selivanov, Yu. A.: Stable and unstable plasma perturbations in the ionospheric $\mathrm{F}$ region, caused by spatial packet of atmospheric gravity waves, Phys. Chem. Earth, 34, 508-515, 2009.

Rudenko, O.: Nonlinear sawtooth-shaped waves, Phys.-Uspekhi, 38, 965-989, 1995.

Soroka, S. A., Kalita, B. I., Karatayeva, L. M., Mezentcev, V. P., Nogach, R. T., and Parrot, M.: Active acoustic experiments with the satellite DEMETER, International Conference DEMETER, Toulouse, 14-16 June 2006, 2006.

Sorokin, V. and Hayakawa, M.: Generation of seismic-related DC electric fields and Lithosphere-Atrmosphere-Ionosphere Coupling, Modern. Appl. Sci., 7, 1-25, 2013.

Sorokin, V. and Hayakawa M.: Plasma and electromagnetic effects caused by the seismic-related disturbances of electric current in the global circuit, Modern Appl. Sci., 8, 61-83, 2014.

Waerden, B. L.: Mathematische Statistik, Springer, Berlin, Heidelberg, New York, 359 pp., 1971.

Xia, Y., Liu, J. Y. T., Cui, X., Li, J., Chen, W., and Liu, C.: Abnormal infrasound signals before $92 M \geqq 7.0$ worldwide earthquakes during 2002-2008, J. Asian Earth Sci., 41, 434-441, 2011.

Zettergren, M. D. and Snively, J. B.: Ionospheric signatures of acoustic waves generated by transient tropospheric forcing, Geophys. Res. Lett., 40, 5345-5349, doi:10.1002/2013GL058018, 2013.

Zettergren, M. D. and Snively, J. B.: Ionospheric response to infrasonic-acoustic waves generated by natural hazard events, J. Geophys. Res., 120, 8002-8024, doi:10.1002/2015JA021116, 2015. 